\title{
Gaussian Sum-Rules and Prediction of Resonance Properties
}

\author{
G. Orlandini, T.G. Steele * \\ Dipartimento di Fisica and \\ INFN Gruppo Collegato di Trento \\ Università di Trento \\ I-38050 Povo, Italy \\ D. Harnett \\ Department of Physics \& Engineering Physics \\ University of Saskatchewan \\ Saskatoon, Saskatchewan S7N 5E2, Canada.
}

October 26, 2018

\begin{abstract}
Techniques for using Gaussian QCD sum-rules to predict hadronic resonance properties are developed for single-resonance and two-resonance phenomenological models, and criteria are developed for determining which of these models is required for analyzing a particular hadronic channel. The vector current sum-rule coupled to the $\rho$ meson is shown to be consistent with a single resonance model, and the Gaussian sum-rule analysis results in an accurate $\rho$ mass prediction which exhibits excellent agreement between the theoretical prediction of the Gaussian sum-rule and the phenomenological model. A two-resonance model is shown to be necessary for the Gaussian sum-rule for the non-strange quark scalar $(\bar{n} n)$ currents. The two-resonance Gaussian sum-rule analysis of the isoscalar and isovector $(I=0,1) \bar{n} n$ scalar mesons exhibits excellent agreement between the theoretical prediction and phenomenological model. The prediction of the resonance properties of the $I=0,1$ $\bar{n} n$ scalar mesons in this two-resonance model provides valuable information for the interpretation of the scalar mesons, including the $X(1775)$.
\end{abstract}

\section{Introduction}

QCD sum-rules are an established technique for relating hadronic properties to theoretical QCD predictions. The most frequently used sum-rules for this purpose are the Laplace [1] and finite-energy sum-rules [2]. The significance of using the finite-energy sum-rule (FESR) as a supplementary constraint on a Laplace sum-rule analysis was most clearly established through the Gaussian sum-rules [3]

$$
G(\hat{s}, \tau)=\frac{1}{\pi} \int_{t_{0}}^{\infty} \frac{1}{\sqrt{4 \pi \tau}} \exp \left(-\frac{(t-\hat{s})^{2}}{4 \tau}\right) \rho(t) d t \quad, \quad \tau>0
$$

where $\rho(t)$ is a hadronic spectral function with physical threshold $t_{0}$. The quantity $G(\hat{s}, \tau)$ on the left-hand side of (11) is determined from a theoretical calculation of a correlation function $\Pi\left(Q^{2}\right)$. The composite operators used to construct the correlation function serve as interpolating fields for the hadronic channel represented by $\rho(t)$ on the right-hand side of (11).

${ }^{*}$ Permanent Address: Department of Physics \& Engineering Physics, University of Saskatchewan, Saskatoon, SK S7N 5E2, Canada 
The Gaussian kernel in (11) implies that $G(\hat{s}, \tau)$ satisfies a diffusion equation [3].

$$
\frac{\partial^{2} G(\hat{s}, \tau)}{\partial \hat{s}^{2}}=\frac{\partial G(\hat{s}, \tau)}{\partial \tau}
$$

The relation between Gaussian and finite-energy sum-rules was established [3] by showing that the resonance plus continuum model for $\rho(t)$, when evolved through the diffusion equation, would only reproduce the QCD prediction at large energies (large $\tau$ ) if the resonance and continuum threshold $s_{0}$ were related by the $n=0$ member of the following FESR family.

$$
F_{n}\left(s_{0}\right)=\frac{1}{\pi} \int_{t_{0}}^{s_{0}} t^{n} \rho(t) d t \quad, \quad n=0,1,2, \ldots
$$

As with the Gaussian sum-rule (17), the left-hand side of (3) is obtained from a QCD prediction.

Sum-rules with Gaussian-like (Lorentzian) kernels have already proven to have strong predictive power in nuclear physics [A]. The purpose of this paper is to demonstrate that Gaussian sum-rules can also be used predictively in hadronic physics, and to develop techniques for the analysis of Gaussian sum-rules in hadronic physics. Section 2 reviews the formulation and conceptual advantages of Gaussian sum-rules, and develops techniques for analyzing a single-resonance plus continuum phenomenological model. Section 3 applies these techniques to the $\rho$ meson which has traditionally been used to establish the validity of sum-rule techniques. Finally in Section 4 we study the phenomenologically challenging case of the quark scalar mesons probed through the non-strange $\bar{n} n$ current. The scalar sector requires an extension of the analysis techniques to a two-resonance phenomenological model, and development of criteria to determine when such an extension is necessary. This sensitivity of the Gaussian sum-rule to excited states is one of the unique features of the Gaussian sum-rule compared with Laplace sum-rules. The phenomenological implications of the results of the Gaussian sum-rule analysis of the $\bar{n} n$ quark scalar mesons will be presented at the end of Section 4 .

\section{Conceptual Foundations of Gaussian Sum-Rules}

Consider the basic form of the Gaussian sum-rule (1). Leaving aside the method for calculating $G(\hat{s}, \tau)$, consider the $\tau \rightarrow 0$ limit of the sum-rule. Using the identity

$$
\lim _{\tau \rightarrow 0} \frac{1}{\sqrt{4 \pi \tau}} \exp \left(-\frac{(t-\hat{s})^{2}}{4 \tau}\right)=\delta(t-\hat{s})
$$

we see that

$$
\lim _{\tau \rightarrow 0} G(\hat{s}, \tau)=\frac{1}{\pi} \rho(\hat{s}) \quad, \quad \hat{s}>t_{0}
$$

Since $\rho(t)$ is related to hadronic physics quantities, the $\tau \rightarrow 0$ limit of the Gaussian sum-rule would in principle allow direct extraction of hadronic physics from the $\hat{s}$ dependence of $G(\hat{s}, \tau)$ obtained from QCD.

Although the above limit cannot be achieved in practice, the property (5) demonstrates an important conceptual advantage of the Gaussian sum-rule. Consider the equivalent form of the Laplace sum-rule

$$
R\left(\Delta^{2}\right)=\frac{1}{\pi} \int_{t_{0}}^{\infty} \exp \left(-\frac{t}{\Delta^{2}}\right) \rho(t) d t .
$$

The $\Delta \rightarrow 0$ limit (again not possible to achieve in practice) would in principle only emphasize the $t=0$ region of the integration region, and would not reveal the energy dependence of $\rho(t)$.

Now consider a more realistic case of a Gaussian sum-rule with $\tau>0$. The Gaussian kernel is peaked at $t=\hat{s}$, and has a width of $\sqrt{2 \tau}$. Thus $G(\hat{s}, \tau)$ represents a smearing of $\rho(t)$ in the region $\hat{s}-\sqrt{2 \tau}<t<\hat{s}+\sqrt{2 \tau}$, centered 
at $t=\hat{s}$. It is therefore reasonable to hope that with $\tau$ fixed at a reasonable physical value, the $\hat{s}$ dependence of $G(\hat{s}, \tau)$ will reveal the rough structure of $\rho(t)$. For example, if $t=m_{r}^{2}$ corresponds to a sharp resonance peak of $\rho(t)$, then one would also expect a peak in $G(\hat{s}, \tau)$ near $\hat{s}=m_{r}^{2}$. These expectations are upheld by the analysis of the $\rho$ and scalar quark mesons in subsequent sections.

The concept of quark-hadron duality, where averaged hadronic quantities are equivalent to the QCD prediction, is explicitly manifested in the parameter $\tau$ which controls the size of the region near $t=\hat{s}$ over which hadronic physics is averaged. As will be discussed below, $\tau$ is directly related to the energy scale for running quantities in the renormalization-group improvement of the theoretical prediction $G(\hat{s}, \tau)$, and no constraint on the parameter $\hat{s}$ emerges. The limitations of QCD originating from the renormalization scale then provide a natural duality interval for reasonable agreement between hadronic physics and QCD. Finally, we shall see that nonperturbative contributions to $G(\hat{s}, \tau)$ are suppressed with increasing $\hat{s}$, explicitly reinforcing the importance of non-perturbative effects in the low-energy region.

The above aspects of the relation between QCD and hadronic physics illuminated by Gaussian sum-rules are obscured in the Laplace sum-rules. The reason for this contrast is that the Laplace kernel exp $\left(-t / \Delta^{2}\right)$ exponentially suppresses the entire energy region of $\rho(t)$, while the Gaussian kernel only damps the energy region of $\rho(t)$ away from $t=\hat{s}$. Thus the two scales $\hat{s}$ and $\tau$ in the Gaussian sum-rule provide a more detailed probe of hadronic physics than the Laplace sum-rule.

\subsection{Single-Resonance Analysis of Gaussian Sum-Rules}

Extraction of hadronic properties from the theoretical prediction $G(\hat{s}, \tau)$ requires a phenomenological model for $\rho(t)$. The "resonance plus continuum" model is used to represent the hadronic physics phenomenology contained in the integral of $\rho(t)$. In this model, hadronic physics is (locally) dual to the theoretical QCD prediction for energies above the continuum threshold $t=s_{0}$

$$
\rho(t)=\theta\left(s_{0}-t\right) \rho^{h a d}(t)+\theta\left(t-s_{0}\right) \operatorname{Im} \Pi^{Q C D}(t)
$$

where $s_{0}$ is the continuum threshold above which hadronic physics and QCD are locally dual. The continuum contribution to the integral of (1) is denoted by

$$
G^{\text {cont }}\left(\hat{s}, \tau, s_{0}\right)=\frac{1}{\pi} \int_{s_{0}}^{\infty} \frac{1}{\sqrt{4 \pi \tau}} \exp \left(-\frac{(t-\hat{s})^{2}}{4 \tau}\right) \operatorname{Im} \Pi^{Q C D}(t) d t
$$

Since the continuum contribution is determined by QCD, it is combined with the theoretical quantity $G(\hat{s}, \tau)$

$$
G^{Q C D}\left(\hat{s}, \tau, s_{0}\right) \equiv G(\hat{s}, \tau)-G^{c o n t}\left(\hat{s}, \tau, s_{0}\right)
$$

resulting in a Gaussian sum-rule relating QCD to hadronic physics phenomenology.

$$
G^{Q C D}\left(\hat{s}, \tau, s_{0}\right)=\frac{1}{\pi} \int_{t_{0}}^{s_{0}} \frac{1}{\sqrt{4 \pi \tau}} \exp \left(-\frac{(t-\hat{s})^{2}}{4 \tau}\right) \rho^{h a d}(t) d t
$$

Consider the single narrow resonance model for $\rho^{\text {had }}(t)$ widely (but not exclusively) employed in the analysis of Laplace sum-rules

$$
\frac{1}{\pi} \rho^{h a d}(t)=f_{r}^{2} \delta\left(t-m_{r}^{2}\right)
$$

where $m_{r}$ denotes the resonance mass, and $f_{r}^{2}$ is the integrated resonance strength. Use of this model in (10) leads to a Gaussian sum-rule which relates QCD to the properties of the single resonance.

$$
G^{Q C D}\left(\hat{s}, \tau, s_{0}\right)=\frac{f_{r}^{2}}{\sqrt{4 \pi \tau}} \exp \left[-\frac{\left(\hat{s}-m_{r}^{2}\right)^{2}}{4 \tau}\right]
$$


The continuum threshold $s_{0}$ is constrained by the finite-energy sum rule (3), which in the single narrow resonance model becomes

$$
F_{0}\left(s_{0}\right)=f_{r}^{2}
$$

Since the finite-energy sum-rule must be satisfied for the Gaussian sum-rule to evolve asymptotically to the QCD prediction through the diffusion equation (2) (heat-evolution test [3]), the normalization of the sum-rule (9) is fixed by the finite-energy sum-rule constraint, as can be verified by taking the $\hat{s}$ integral of (12).

$$
\int_{-\infty}^{\infty} G^{Q C D}\left(\hat{s}, \tau, s_{0}\right) d \hat{s}=f_{r}^{2}
$$

Thus since the quantity $f_{r}^{2}$ is constrained by the finite-energy sum-rule (13), the normalization of the quantity $G^{Q C D}\left(\hat{s}, \tau, s_{0}\right)$ is already determined. Consequently, the information in the sum-rule (12) remaining after imposing the heat-evolution test [3] is contained in the quantity $N^{Q C D}\left(\hat{s}, \tau, s_{0}\right)$ normalized to unit area

$$
\begin{aligned}
& N^{Q C D}\left(\hat{s}, \tau, s_{0}\right)=\frac{G^{Q C D}\left(\hat{s}, \tau, s_{0}\right)}{M_{0}\left(\tau, s_{0}\right)} \\
& M_{0}\left(\tau, s_{0}\right)=\int_{-\infty}^{\infty} G^{Q C D}\left(\hat{s}, \tau, s_{0}\right) d \hat{s},
\end{aligned}
$$

leading to a sum-rule independent of the resonance-strength and finite-energy sum-rule.

$$
N^{Q C D}\left(\hat{s}, \tau, s_{0}\right)=\frac{1}{\sqrt{4 \pi \tau}} \exp \left[-\frac{\left(\hat{s}-m_{r}^{2}\right)^{2}}{4 \tau}\right]
$$

Viewed as a function of $\hat{s}$, the phenomenological (right-hand) side of (17) has a peak (maximum) which occurs at $\hat{s}=m_{r}^{2}$ independent of $\tau$, and the peak height $1 / \sqrt{4 \pi \tau}$ at this maximum shows $\tau$ dependence. These properties will be used to find the optimum value of $s_{0}$ from which $m_{r}^{2}$ can be extracted. This optimum value of $s_{0}$ can then be used to determine the total resonance strength through the finite-energy sum-rule (13).

The analysis proceeds by fixing $\tau$, and finding the value $\hat{s}_{\text {peak }}\left(\tau, s_{0}\right)$ at which $N^{Q C D}\left(\hat{s}, \tau, s_{0}\right)$ has a maximum!

$$
\left.\frac{d}{d \hat{s}} N^{Q C D}\left(\hat{s}, \tau, s_{0}\right)\right|_{\hat{s}=\hat{s}_{p e a k}\left(\tau, s_{0}\right)}=0
$$

and then determining the peak height

$$
N_{\text {peak }}\left(\tau, s_{0}\right)=N^{Q C D}\left(\hat{s}_{\text {peak }}\left(\tau, s_{0}\right), \tau, s_{0}\right) \quad .
$$

The optimum value of $s_{0}$ is constrained by the requirements following from the properties of the phenomenological side of (17):

1. $\hat{s}_{\text {peak }}\left(\tau, s_{0}\right)=m_{r}^{2}$ is constant $(\tau$-independent $)$,

2. $\sqrt{4 \pi \tau} N_{\text {peak }}\left(\tau, s_{0}\right)=1$ (independent of $\tau$ ).

Thus the optimum value of $s_{0}$, and the corresponding prediction of the resonance mass $m_{r}^{2}$, can be determined by minimizing a $\chi^{2}$ measure related to the above properties

$$
\chi^{2}\left(s_{0}\right)=\sum_{i=1}^{n}\left(\frac{\hat{s}_{\text {peak }}\left(\tau_{i}, s_{0}\right)}{m_{r}^{2}}-1\right)^{2}+\sum_{i=1}^{n}\left(N_{\text {peak }}\left(\tau_{i}, s_{0}\right) \sqrt{4 \pi \tau_{i}}-1\right)^{2}
$$

\footnotetext{
${ }^{1}$ Our numerical analysis uses the Golden search algorithm 5 to determine $\hat{s}_{\text {peak }}\left(\tau, s_{0}\right)$
} 
where $\tau_{i}$ are equally-spaced points in the $\tau$ region of interest, and $m_{r}^{2}$ is implicitly a function of $s_{0}$

$$
m_{r}^{2}=\frac{1}{n} \sum_{i=1}^{n} \hat{s}_{\text {peak }}\left(\tau_{i}, s_{0}\right)
$$

The value of $s_{0}$ which minimizes the $\chi^{2}$ thus provides the best agreement with properties of the phenomenological model and leads to a prediction of the resonance mass $m_{r}^{2}$ in the single narrow-resonance model.

Before proceeding with the application of these $\chi^{2}$ techniques to the $\rho$ meson, extensions of the narrow resonance model will be discussed to determine the magnitude of effects that could arise from resonance widths $\Gamma$ and resonance shapes. Intuitively, one would expect that if the Gaussian width is much wider than the resonance width, then the width effects will be negligible.

The simplest extension of the unit-area narrow resonance $\delta\left(t-m_{r}^{2}\right)$ is the unit area square pulse previously used for studying width effects in the Laplace sum-rules [6]

$$
\frac{1}{\pi} \rho^{s p}(t)=\frac{1}{2 m_{r} \Gamma}\left[\theta\left(t-m_{R}^{2}+m_{r} \Gamma\right)-\theta\left(t-m_{R}^{2}-m_{r} \Gamma\right)\right],
$$

which has the following Gaussian image

$$
\begin{aligned}
G^{s p}(\hat{s}, \tau) & =\frac{1}{2 m_{r} \Gamma \sqrt{4 \pi \tau}} \int_{m_{r}^{2}-m_{r} \Gamma}^{m_{r}^{2}+m_{r} \Gamma} \exp \left(-\frac{(t-\hat{s})^{2}}{4 \tau}\right) d t \\
& =\frac{1}{4 m_{r} \Gamma}\left[\operatorname{erf}\left(\frac{\hat{s}-m_{R}^{2}+m_{r} \Gamma}{2 \sqrt{\tau}}\right)-\operatorname{erf}\left(\frac{\hat{s}-m_{R}^{2}-m_{r} \Gamma}{2 \sqrt{\tau}}\right)\right],
\end{aligned}
$$

where

$$
\operatorname{erf}(x)=\frac{2}{\sqrt{\pi}} \int_{0}^{x} e^{-y^{2}} d y .
$$

Such a resonance model clearly overestimates the effect of resonance widths compared with a Breit-Wigner which is more concentrated about the resonance peak. The expression (23) still has a peak position at $\hat{s}=m_{r}^{2}$ as in the narrow resonance model, but its peak height is altered to

$$
G^{s p}\left(\hat{s}=m_{r}^{2}, \tau\right)=\frac{1}{2 m_{r} \Gamma} \operatorname{erf}\left(\frac{m_{r} \Gamma}{2 \sqrt{\tau}}\right) \approx \frac{1}{\sqrt{4 \pi \tau}}\left[1-\frac{m_{r}^{2} \Gamma^{2}}{12 \tau}+\mathcal{O}\left(\Gamma^{4}\right)\right]
$$

As anticipated, the resonance width effects in (25) diminish with increasing Gaussian width $\tau$. Using the full expression in terms of the error function, the deviation from the narrow width limit is less than $5 \%$ for $m_{r} \Gamma /(2 \sqrt{\tau})<$ 0.4 , a condition which is easily satisfied for even a wide resonance such as the $f_{0}(1370)$ in the $\tau>1 \mathrm{GeV}^{4}$ range. Such uncertainties are well below those associated with the theoretical prediction of $G(\hat{s}, \tau)$. For the $\rho$ meson, the resonance width is a completely negligible $0.2 \%$ effect even for $\tau=0.5 \mathrm{GeV}^{4}$. Thus we conclude that for reasonable ranges of the the Gaussian width $\tau$, the Gaussian sum-rule is insensitive to the effect of resonance widths, and so the narrow width phenomenological model is in fact an accurate description of non-zero width resonances in the Gaussian sum-rules.

\subsection{Formulation of Gaussian Sum-Rules}

In this section we briefly summarize salient features of the formulation of Gaussian sum-rules presented in [3]. Consider a dispersion relation with one subtraction constant as needed for the correlation function of vector currents 
used to probe the $\rho$ meson 2 .

$$
\Pi\left(Q^{2}\right)=\Pi(0)-\frac{Q^{2}}{\pi} \int_{t_{0}}^{\infty} d t \frac{\rho(t)}{t\left(t+Q^{2}\right)}
$$

The difference between the dispersion relation at $Q^{2}=-\hat{s}+i \Delta$ and $Q^{2}=-\hat{s}-i \Delta$ cancels the dependence on the subtraction constant $\Pi(0)$.

$$
\frac{\Pi(-\hat{s}-i \Delta)-\Pi(-\hat{s}+i \Delta)}{2 i \Delta}=\frac{1}{\pi} \int_{t_{0}}^{\infty} d t \frac{\rho(t)}{(t-\hat{s})^{2}+\Delta^{2}}
$$

For large $\Delta$ the left-hand side of (27) is determined by QCD since it evaluates the correlation function well away from the physical cut. As with Laplace sum-rules [1], the Gaussian sum-rule can be obtained from the dispersion relation (27) through the Borel transform operator $B$

$$
\hat{B} \equiv \lim _{\substack{N \rightarrow \infty \\ \Delta^{2} / N \equiv 4 \tau}} \frac{\left(-\Delta^{2}\right)^{N}}{\Gamma(N)}\left(\frac{d}{d \Delta^{2}}\right)^{N}
$$

which has the following property relevant to construction of the Gaussian sum-rule

$$
\hat{B}\left[\frac{1}{x+\Delta^{2}}\right]=\frac{1}{4 \tau} \exp \left(-\frac{x}{4 \tau}\right) .
$$

Defining the theoretically-determined quantity

$$
G(\hat{s}, \tau)=\sqrt{\frac{\tau}{\pi}} \hat{B}\left[\frac{\Pi(-\hat{s}-i \Delta)-\Pi(-\hat{s}+i \Delta)}{i \Delta}\right]
$$

leads to the Gaussian sum-rule (11) after application of $\hat{B}$ to (27).

An alternative to the direct calculation of the Gaussian sum-rules through the definition of $\hat{B}$ in (28) is obtained through an identity relating the Borel and Laplace transform [3].

$$
\begin{aligned}
& f\left(\Delta^{2}\right)=\int_{0}^{\infty} F(T) e^{-\Delta^{2} T} d T \equiv \mathcal{L}[F(T)] \Longrightarrow \frac{1}{T} \hat{B}\left[f\left(\Delta^{2}\right)\right]=F(T)=\mathcal{L}^{-1}\left[f\left(\Delta^{2}\right)\right] \quad, \quad T \equiv \frac{1}{4 \tau} \\
& \mathcal{L}^{-1}\left[f\left(\Delta^{2}\right)\right]=\frac{1}{2 \pi i} \int_{b-i \infty}^{b+i \infty} f\left(\Delta^{2}\right) e^{\Delta^{2} T} d \Delta^{2}
\end{aligned}
$$

where the real parameter $b$ in the definition (32) of the inverse Laplace transform must be chosen so that $f\left(\Delta^{2}\right)$ is analytic to the right of the contour of integration in the complex $\Delta^{2}$ plane. Using the result (31), the Gaussian sum-rule (30) can be obtained from an inverse Laplace transform

$$
\begin{aligned}
G(\hat{s}, \tau) & =\frac{1}{4 \sqrt{\pi \tau}} \mathcal{L}^{-1}\left[\frac{\Pi(-\hat{s}-i \Delta)-\Pi(-\hat{s}+i \Delta)}{i \Delta}\right] \\
& =\frac{1}{4 \sqrt{\pi \tau}} \frac{1}{2 \pi i} \int_{b-i \infty}^{b+i \infty} \frac{\Pi(-\hat{s}-i \Delta)-\Pi(-\hat{s}+i \Delta)}{i \Delta} \exp \left(\Delta^{2} T\right) d \Delta^{2}
\end{aligned}
$$

\footnotetext{
${ }^{2}$ Extension to correlation functions requiring further subtraction constants leads to a final result identical to (30) obtained for a single subtraction constant.
} 
Respectively mapping the individual terms in (33) containing $\Pi(-\hat{s} \pm i \Delta)$ from the $\Delta^{2}$ to the $w=-\hat{s} \pm i \Delta$ complex plane results in the following expression for the Gaussian sum-rule

$$
G(\hat{s}, \tau)=\frac{1}{\sqrt{4 \pi \tau}} \frac{1}{2 \pi i} \int_{\Gamma_{1}+\Gamma_{2}} \Pi(w) \exp \left[-\frac{(w+\hat{s})^{2}}{4 \tau}\right] d w
$$

where the contours $\Gamma_{1}$ and $\Gamma_{2}$ are illustrated in Figure 1.

\section{Prediction of the $\rho$ Mass from Gaussian Sum-Rules}

The $\rho$ meson is probed through the vector-isovector current correlation function.

$$
\begin{aligned}
& \Pi_{v}\left(Q^{2}\right)\left[q^{\mu} q^{\nu}-q^{2} g^{\mu \nu}\right]=i \int d^{4} x e^{i q \cdot x}\left\langle O\left|T\left[J^{\mu}(x) J^{\nu}(0)\right]\right| O\right\rangle \quad, \quad Q^{2}=-q^{2}>0 \\
& J^{\mu}(x)=\frac{1}{2}\left[\bar{u}(x) \gamma^{\mu} u(x)-\bar{d}(x) \gamma^{\mu} d(x)\right]
\end{aligned}
$$

The field-theoretical (QCD) calculation of $\Pi_{v}\left(Q^{2}\right)$ consists of perturbative (logarithmic) corrections and QCD vacuum effects of infinite correlation length parametrized by the power-law corrections from the QCD vacuum condensates [1]

$$
\Pi_{v}\left(Q^{2}\right)=\Pi_{v}^{\text {pert }}\left(Q^{2}\right)+\Pi_{v}^{\text {cond }}\left(Q^{2}\right)
$$

To two-loop order in the chiral limit $m_{u}=m_{d}=0$ the perturbative contribution $\beta$ in the $\overline{\mathrm{MS}}$ scheme for three active flavours is [7]

$$
\Pi_{v}^{\text {pert }}\left(Q^{2}\right)=-\frac{1}{8 \pi^{2}} \log \left(\frac{Q^{2}}{\nu^{2}}\right)\left[1+\frac{\alpha(\nu)}{\pi}\right]
$$

where $\nu$ is the renormalization scale. To lowest order, the QCD condensate contributions up to dimension 8 are given by [1]

$$
\begin{aligned}
& \Pi_{v}^{\text {cond }}\left(Q^{2}\right)=\frac{1}{8 \pi^{2}}\left[\frac{1}{Q^{4}}\left\langle C_{4}^{v} \mathcal{O}_{4}^{v}\right\rangle+\frac{1}{Q^{6}}\left\langle C_{6}^{v} \mathcal{O}_{6}^{v}\right\rangle+\frac{1}{Q^{8}}\left\langle C_{8}^{v} \mathcal{O}_{8}^{v}\right\rangle\right] \\
& \left\langle C_{4}^{v} \mathcal{O}_{4}^{v}\right\rangle=\frac{\pi}{3}\left\langle\alpha G^{2}\right\rangle-8 \pi^{2} m\langle\bar{q} q\rangle \\
& \left\langle C_{6}^{v} \mathcal{O}_{6}^{v}\right\rangle=-\frac{896}{81} \pi^{3} \alpha(\langle\bar{q} q\rangle)^{2}
\end{aligned}
$$

where $S U(2)$ symmetry and the vacuum saturation hypothesis have been employed. For brevity, we refer to the literature [8] for the expressions for the dimension eight condensates, and simply use (39) to establish a normalization consistent with [9].

To evaluate the Gaussian sum-rule consider the contour $C(R)$ in Figure 2. The quantity $\Pi(w)$ is analytic within and on $C(R)$; consequently

$$
0=\frac{1}{\sqrt{4 \pi \tau}} \frac{1}{2 \pi i} \oint_{C(R)} \Pi(w) \exp \left[-\frac{(w+\hat{s})^{2}}{4 \tau}\right] d w .
$$

In the limit as $R \rightarrow \infty$, the integrals along $\Gamma_{3}, \Gamma_{4}$ and $\Gamma_{5}$ all approach zero for the QCD expressions given in 38, 39). Furthermore $\tilde{\Gamma}_{1}(R)$ and $\tilde{\Gamma}_{1}(R)$ respectively approach $\Gamma_{1}$ and $\Gamma_{2}$ as $R \rightarrow \infty$. We therefore obtain the

\footnotetext{
${ }^{3}$ A divergent constant has been ignored since like the subtraction constant $\Pi(0)$, it vanishes in the formation of the Gaussian sum rule.
} 
following expression for the Gaussian sum-rule (34)

$$
\begin{aligned}
G(\hat{s}, \tau)= & -\frac{1}{\sqrt{4 \pi \tau}} \frac{1}{2 \pi i} \lim _{R \rightarrow \infty} \int_{\Gamma_{c}+\Gamma_{\epsilon}} \Pi(w) \exp \left[-\frac{(w+\hat{s})^{2}}{4 \tau}\right] d w \\
= & -\frac{1}{\sqrt{4 \pi \tau}} \frac{1}{2 \pi i} \int_{\epsilon}^{\infty}\left[\Pi\left(t e^{i \pi}\right)-\Pi\left(t e^{-i \pi}\right)\right] \exp \left(-\frac{(t-\hat{s})^{2}}{4 \tau}\right) d t \\
& +\frac{1}{\sqrt{4 \pi \tau}} \frac{1}{2 \pi} \int_{-\pi}^{\pi} \epsilon e^{i \theta} \Pi\left(\epsilon e^{i \theta}\right) \exp \left(-\frac{\left(\epsilon e^{i \theta}+\hat{s}\right)^{2}}{4 \tau}\right) d \theta .
\end{aligned}
$$

The perturbative contributions from the $\Gamma_{\epsilon} \operatorname{contour~(~} \theta$ integral) in (43) will be zero in the $\epsilon \rightarrow 0$ limit, leaving only the integral of the discontinuity across the branch cut $\left[i . e\right.$. $\left.\operatorname{Im} \Pi^{\text {pert }}(t)\right]$ to determine the perturbative contributions to the Gaussian sum-rule

$$
G^{\text {pert }}(\hat{s}, \tau)=\frac{1}{\sqrt{4 \pi \tau}} \int_{0}^{\infty} \frac{1}{8 \pi^{2}}\left(1+\frac{\alpha(\nu)}{\pi}\right) \exp \left(-\frac{(t-\hat{s})^{2}}{4 \tau}\right) d t=\frac{1}{16 \pi^{2}}\left(1+\frac{\alpha(\nu)}{\pi}\right)\left[1+\operatorname{erf}\left(\frac{\hat{s}}{2 \sqrt{\tau}}\right)\right],
$$

Since the current (36) is renormalization-group invariant, $\operatorname{Im} \Pi^{\text {pert }}(t)$ satisfies a homogeneous renormalization-group equation. The result of renormalization-group improvement for the Gaussian sum-rules can be inferred from the general structure of perturbative corrections which take the form

$$
\int_{0}^{\infty} \log ^{n}\left(\frac{t}{\nu^{2}}\right) \exp \left(-\frac{(t-\hat{s})^{2}}{4 \tau}\right) d t=\nu^{2} \int_{0}^{\infty} d x \log ^{n}(x) \exp \left[-\left(\frac{\nu^{2} x}{2 \sqrt{\tau}}-\frac{\hat{s}}{2 \sqrt{\tau}}\right)^{2}\right]=\nu^{2} H\left(\frac{\nu^{2}}{\sqrt{\tau}}, \frac{\hat{s}}{\sqrt{\tau}}\right) .
$$

The functional dependence of the perturbative corrections to the Gaussian sum-rules expressed by the function $H$ demonstrates that $\nu$ scales with $\sqrt{\tau}$. Thus, the solution of the renormalization-group equation satisfied by the perturbative contributions is the replacement of $\alpha(\nu)$ with the running coupling constant and identifies the scale $\nu^{2}=\sqrt{\tau}$. This implies the existence of low-energy boundary (lower bound) on $\tau$, but places no restriction on the scale $\hat{s}$.

The QCD condensate contributions (39) to $\Pi\left(Q^{2}\right)$ do not have a branch discontinuity, so their contribution to the Gaussian sum-rule arises solely from the contour $\Gamma_{\epsilon}(\theta$ integral $)$ in (43), and can be evaluated using the result

$$
-\frac{1}{2 \pi i} \int_{\Gamma_{\epsilon}} \frac{1}{w^{n}} \exp \left[-\frac{(w+\hat{s})^{2}}{4 \tau}\right] d w=\lim _{w \rightarrow 0} \frac{1}{(n-1) !} \frac{d^{n-1}}{d w^{n-1}} \exp \left[-\frac{(w+\hat{s})^{2}}{4 \tau}\right] \quad, \quad n=1,2,3 \ldots \quad .
$$

Expressions (44), (46), (39), and (43) lead to the Gaussian sum-rule of vector currents.

$$
\begin{aligned}
G(\hat{s}, \tau)= & \frac{1}{16 \pi^{2}}\left(1+\frac{\alpha(\sqrt{\tau})}{\pi}\right)\left[1+\operatorname{erf}\left(\frac{\hat{s}}{2 \sqrt{\tau}}\right)\right]-\frac{\hat{s}}{32 \pi^{2} \tau \sqrt{\pi \tau}} \exp \left(-\frac{\hat{s}^{2}}{4 \tau}\right)\left\langle C_{4}^{v} \mathcal{O}_{4}^{v}\right\rangle \\
& +\frac{1}{64 \pi^{2} \tau \sqrt{\pi \tau}}\left(-1+\frac{\hat{s}^{2}}{2 \tau}\right) \exp \left(-\frac{\hat{s}^{2}}{4 \tau}\right)\left\langle C_{6}^{v} \mathcal{O}_{6}^{v}\right\rangle-\frac{\hat{s}}{128 \pi^{2} \tau^{2} \sqrt{\pi \tau}}\left(-1+\frac{\hat{s}^{2}}{6 \tau}\right) \exp \left(-\frac{\hat{s}^{2}}{4 \tau}\right)\left\langle C_{8}^{v} \mathcal{O}_{8}^{v}\right\rangle
\end{aligned}
$$

Agreement between (47) and [3] provides a useful check on the conventions established in (34). We also note that the QCD condensate contributions to (47) can be recovered by expanding the Gaussian kernel in (11) about $t=0$ (or alternatively in a series about any other value of $t$, or in a series of Hermite polynomials), and then using the relation between the QCD condensates and the FESR family (3) (in addition, see equation (6.15) in reference [3]). This demonstrates that in principle (e.g. knowledge of higher-dimension condensate contributions), sum-rules 
based on the Gaussian (or Laplace) kernel contain the same information as the whole FESR family. However, as we discussed previously and will be demonstrated below, the Gaussian kernel arranges this information more effectively, enhancing the predictive power compared with other sum-rule kernels.

The QCD continuum contributions (8) arising from the perturbative corrections to the vector correlator are

$$
G^{c o n t}\left(\hat{s}, \tau, s_{0}\right)=\frac{1}{16 \pi^{2}}\left(1+\frac{\alpha(\sqrt{\tau})}{\pi}\right)\left[1-\operatorname{erf}\left(\frac{s_{0}-\hat{s}}{2 \sqrt{\tau}}\right)\right]
$$

and hence the theoretically determined Gaussian sum-rule quantity (9) for the vector currents is

$$
\begin{aligned}
G^{Q C D}\left(\hat{s}, \tau, s_{0}\right)= & \frac{1}{16 \pi^{2}}\left(1+\frac{\alpha(\sqrt{\tau})}{\pi}\right)\left[\operatorname{erf}\left(\frac{\hat{s}}{2 \sqrt{\tau}}\right)+\operatorname{erf}\left(\frac{s_{0}-\hat{s}}{2 \sqrt{\tau}}\right)\right]-\frac{\hat{s}}{32 \pi^{2} \tau \sqrt{\pi \tau}} \exp \left(-\frac{\hat{s}^{2}}{4 \tau}\right)\left\langle C_{4}^{v} \mathcal{O}_{4}^{v}\right\rangle \\
& +\frac{1}{64 \pi^{2} \tau \sqrt{\pi \tau}}\left(-1+\frac{\hat{s}^{2}}{2 \tau}\right) \exp \left(-\frac{\hat{s}^{2}}{4 \tau}\right)\left\langle C_{6}^{v} \mathcal{O}_{6}^{v}\right\rangle \\
& -\frac{\hat{s}}{128 \pi^{2} \tau^{2} \sqrt{\pi \tau}}\left(-1+\frac{\hat{s}^{2}}{6 \tau}\right) \exp \left(-\frac{\hat{s}^{2}}{4 \tau}\right)\left\langle C_{8}^{v} \mathcal{O}_{8}^{v}\right\rangle .
\end{aligned}
$$

Finally, the quantity $M_{0}\left(\tau, s_{0}\right)$ in (16) required for the normalized vector-current Gaussian sum-rule (15) is given by

$$
M_{0}\left(\tau, s_{0}\right)=\int_{-\infty}^{\infty} G^{Q C D}\left(\hat{s}, \tau, s_{0}\right) d \hat{s}=\frac{1}{8 \pi^{2}}\left(1+\frac{\alpha(\sqrt{\tau})}{\pi}\right) s_{0}
$$

The non-perturbative QCD condensate contributions in (49) are exponentially suppressed for large $\hat{s}$. Since $\hat{s}$ represents the location of the Gaussian peak on the phenomenological side of the sum-rule, the non-perturbative corrections are most important in the low-energy region, as anticipated by the role of QCD condensates in relation to the vacuum properties of QCD. This explicit low-energy role of the QCD condensates clearly exhibited for the Gaussian sum-rules is obscured in the Laplace sum-rule because the peak of the Laplace exponential kernel in (6) is always located at $t=0$.

Several QCD parameters must be specified before carrying out the phenomenological analysis of the Gaussian sum-rule of vector currents. The running coupling for three active flavours to two-loop order is

$$
\frac{\alpha_{s}\left(\nu^{2}\right)}{\pi}=\frac{1}{\beta_{0} L}-\frac{\beta_{1} \log L}{\beta_{0}\left(\beta_{0} L\right)^{2}} \quad, \quad L=\log \left(\frac{\nu^{2}}{\Lambda^{2}}\right) \quad, \quad \beta_{0}=\frac{9}{4} \quad, \quad \beta_{1}=4
$$

with $\Lambda_{\overline{M S}} \approx 300 \mathrm{MeV}$ for three active flavours, consistent with current estimates of $\alpha_{s}\left(M_{\tau}\right)$ [10, 11] and matching conditions through the charm threshold [12]. For the gluon condensate we use the central value determined in [9]

$$
\left\langle\alpha G^{2}\right\rangle=(0.045 \pm 0.014) \mathrm{GeV}^{4},
$$

and PCAC [13] is used for the quark condensate.

$$
m\langle\bar{q} q\rangle=\frac{1}{2} m_{u}\langle\bar{u} u\rangle+\frac{1}{2} m_{d}\langle\bar{d} d\rangle=-\frac{1}{2} f_{\pi}^{2} m_{\pi}^{2} \quad, \quad f_{\pi}=93 \mathrm{MeV} \quad .
$$

Deviations of the dimension-six condensate from the vacuum saturation value are parameterized by the quantity $f_{v s}$ which could be as large as $f_{v s}=2$ [9, 14]

$$
\left\langle C_{6}^{v} \mathcal{O}_{6}^{v}\right\rangle=-f_{v s} \frac{896}{81} \pi^{3} \alpha(\langle\bar{q} q\rangle)^{2}=-f_{v s} \frac{896}{81} \pi^{3}\left(1.8 \times 10^{-4} \mathrm{GeV}^{6}\right) \quad, \quad f_{v s}=1.5 \pm 0.5
$$

Finally, we use the reference [9] value for the dimension-eight condensate.

$$
\left\langle C_{8}^{v} \mathcal{O}_{8}^{v}\right\rangle=(0.40 \pm 0.16) \mathrm{GeV}^{8}
$$


The $\chi^{2}$ minimization techniques outlined in Section 2.1 [see equations (20) and 212] are now employed to determine the optimum continuum threshold $s_{0}$ and the corresponding prediction of the $\rho$ meson mass $m_{\rho}$, demonstrating the ability of Gaussian sum-rules to predict resonance properties. Using equally-spaced points in the rangef $0.5 \mathrm{GeV}^{4} \leq \tau \leq 4.0 \mathrm{GeV}^{4}$ for determining $\chi^{2}\left(s_{0}\right)$ in $(20)$, and with inclusion of the uncertainties of the QCD condensates given in (52 55) we obtain the following results for $m_{\rho}$ and $s_{0}$

$$
m_{\rho}=(0.75 \pm 0.07) \mathrm{GeV} \quad, \quad s_{0}=(1.2 \pm 0.2) \mathrm{GeV}^{2}
$$

in excellent agreement with the measured mass $m_{\rho}=770 \mathrm{MeV}$.

A detailed examination of the required equality (17) between the Gaussian sum-rule and the phenomenological single resonance model can be obtained by using the $\chi^{2}$-predicted values of $m_{\rho}$ and $s_{0}$ as input into the singleresonance plus continuum model, and examining the $\hat{s}, \tau$ dependence of the sum-rule in comparison with the phenomenological model. Figure 3 compares this $\hat{s}$ dependence of the sum-rule and phenomenological model for selected $\tau$ values in the region used to define the $\chi^{2}$. As would be anticipated by its construction, the $\chi^{2}$ optimization procedure leads to excellent correspondence between theory and phenomenology for the $\hat{s}_{\text {peak }}$ position and peak height. However, the astounding agreement between the $\hat{s}$ dependence of the sum-rule and phenomenological model provides strong evidence for the ability of Gaussian sum-rules to predict hadronic properties. In the next section, Gaussian sum-rules will be employed to study the (non-strange) $\bar{n} n$ scalar mesons, a more challenging channel for hadronic physics phenomenology.

\section{Gaussian Sum-Rule Analysis of the Quark Scalar Mesons}

The interpretation of the scalar mesons is a challenging problem in hadronic physics since a variety of interpretations exist for the lowest-lying scalar resonances $\left(\sigma\right.$ or $f_{0}(400-1200), f_{0}(980), f_{0}(1370), f_{0}(1500), a_{0}(980), a_{0}(1450)$ [10]) including conventional quark-antiquark $(q \bar{q})$ states, $K \bar{K}$ molecules, gluonium, four-quark models, and dynamically generated thresholds $15,16,17$.

The relevant correlation function for the $I=0,1$ non-strange $\bar{n} n$ scalar mesons is

$$
\Pi_{s}\left(Q^{2}\right)=i \int d^{4} x e^{i q \cdot x}\left\langle 0\left|T\left[J_{s}(x) J_{s}(0)\right]\right| 0\right\rangle \quad, \quad Q^{2}=-q^{2}>0
$$

where, in the $S U(2)$ limit $\left[m_{q} \equiv\left(m_{u}+m_{d}\right) / 2\right]$ for isoscalar $(I=0)$ and isovector $(I=1)$ currents,

$$
J_{s}(x)=m_{q}\left[\bar{u}(x) u(x)+(-1)^{I} \bar{d}(x) d(x)\right] / 2 .
$$

We note that the factor of the quark mass is necessary for a renormalization-group invariant current. Correlation functions of scalar and pseudoscalar currents are unique since they receive significant contributions from instantons [18, 19]. In contrast to QCD condensates which represent vacuum effects of infinite correlation length, instanton contributions represent finite correlation-length QCD vacuum effects, and are the only known theoretical mechanism that distinguishes between the $I=0$ and $I=1$ correlation functions in the presence of $S U(2)$ flavour symmetry [20]. Thus the theoretical calculation of the scalar current correlation function consists of perturbative, condensate, and instanton contributions

$$
\Pi_{s}\left(Q^{2}\right)=\Pi_{s}^{\text {pert }}\left(Q^{2}\right)+\Pi_{s}^{\text {cond }}\left(Q^{2}\right)+\Pi_{s}^{\text {inst }}\left(Q^{2}\right)
$$

At two-loop order and to leading order in the quark mass for three active flavours in the $\overline{M S}$ scheme, the perturbative contributions in (59) are 21]

$$
\Pi_{s}^{\text {pert }}\left(Q^{2}\right)=\frac{3 m_{q}^{2} Q^{2}}{16 \pi^{2}} \log \left(\frac{Q^{2}}{\nu^{2}}\right)\left[1+\frac{\alpha}{\pi}\left(-\log \left(\frac{Q^{2}}{\nu^{2}}\right)+\frac{17}{3}\right)\right] .
$$

\footnotetext{
${ }^{4}$ Altering the range to $1.0 \mathrm{GeV}^{4} \leq \tau \leq 4.0 \mathrm{GeV}^{4}$ has minimal effect on the predictions.

${ }^{5} \mathrm{~A}$ field-theoretical divergence proportional to $Q^{2}$ is ignored since it vanishes after application of $\hat{B}$ in the formation of the Gaussian sum-rule.
} 
To leading order in $m_{q}$ and $\alpha$, the QCD condensate contributions in (59) are 22, 23, 1]:

$$
\begin{aligned}
& \Pi_{s}^{\text {cond }}\left(Q^{2}\right)=m_{q}^{2}\left[\frac{\left\langle C_{4}^{s} \mathcal{O}_{4}^{s}\right\rangle}{Q^{2}}+\frac{\left\langle C_{6}^{s} \mathcal{O}_{6}^{s}\right\rangle}{Q^{4}}\right] \\
& \left\langle C_{4}^{s} \mathcal{O}_{4}^{s}\right\rangle=\frac{3}{2}\left\langle m_{q} \bar{q} q\right\rangle+\frac{1}{16 \pi}\left\langle\alpha_{s} G^{2}\right\rangle \\
& \left\langle C_{6}^{s} \mathcal{O}_{6}^{s}\right\rangle=\pi \alpha_{s}\left[\frac{1}{4}\left\langle\left(\bar{u} \sigma_{\mu \nu} \lambda^{a} u-\bar{d} \sigma_{\mu \nu} \lambda^{a} d\right)^{2}\right\rangle+\frac{1}{6}\left\langle\left(\bar{u} \gamma_{\mu} \lambda^{a} u+\bar{d} \gamma_{\mu} \lambda^{a} d\right) \sum_{u, d, s} \bar{q} \gamma^{\mu} \lambda^{a} q\right\rangle\right]
\end{aligned}
$$

As for the vector current correlation function, the vacuum saturation hypothesis [1] in the $S U(2)$ limit $\langle\bar{u} u\rangle=$ $\langle\bar{d} d\rangle \equiv\langle\bar{q} q\rangle$ provides a reference value for $\left\langle\mathcal{O}_{6}^{s}\right\rangle$

$$
\left\langle C_{6}^{s} \mathcal{O}_{6}^{s}\right\rangle=-f_{v s} \frac{88}{27} \alpha_{s}\left\langle(\bar{q} q)^{2}\right\rangle=-f_{v s} 1.8 \times 10^{-4} \mathrm{GeV}^{6} .
$$

As mentioned earlier, the perturbative and condensate contributions to the scalar correlation function are independent of $I$, and hence do not distinguish between the isoscalar and isovector channels.

Using symmetry properties relating the instanton contributions to pseudoscalar and scalar correlation functions 20] combined with the instanton liquid model results for the pseudoscalar correlation function [1, 19], the instanton contributions to the scalar correlation function are

$$
\Pi_{s}^{\text {inst }}\left(Q^{2}\right)=(-1)^{I} \frac{3 m_{q}^{2} Q^{2}}{4 \pi^{2}}\left[K_{1}\left(\rho_{c} \sqrt{Q^{2}}\right)\right]^{2},
$$

where $K_{1}$ is a modified Bessel function [25], and $\rho_{c}=1 /(600 \mathrm{MeV})$ is the (uniform) instanton size in the instanton liquid model [19]. The explicit factor depending on $I$ in (65) is the only theoretical contribution that distinguishes between the isovector and isoscalar channels.

Calculation of the Gaussian sum-rule for the scalar currents proceeds as in Section 3. Using the following asymptotic property of the modified Bessel function [25]

$$
K_{1}(z) \sim \sqrt{\frac{\pi}{2 z}} e^{-z} ;|z| \gg 1,|\arg (z)| \leq \frac{3 \pi}{2}
$$

one can verify that the QCD expressions given in (60, 61, 65) uphold the transition from (42) to (43) developed for the vector currents. Thus we can calculate the Gaussian sum-rule for scalar currents from (33).

As with the vector channel, the perturbative contributions (60) from the contour $\Gamma_{\epsilon}(\theta$ integral) in (43) will be zero in the $\epsilon \rightarrow 0$ limit, leaving only the integral of the discontinuity across the branch cut $\left[i . e\right.$. $\left.\operatorname{Im} \Pi^{\text {pert }}(t)\right]$ to determine the perturbative contributions to the Gaussian sum-rule.

$$
G^{\text {pert }}(\hat{s}, \tau)=\frac{1}{\sqrt{4 \pi \tau}} \frac{3 m_{q}^{2}(\sqrt{\tau})}{16 \pi^{2}} \int_{0}^{\infty}\left[t\left(1+\frac{17}{3} \frac{\alpha(\sqrt{\tau})}{\pi}\right)-2 \frac{\alpha(\sqrt{\tau})}{\pi} t \log \left(\frac{t}{\sqrt{\tau}}\right)\right] \exp \left(-\frac{(t-\hat{s})^{2}}{4 \tau}\right) d t
$$

This expression has already been renormalization-group improved by the replacement of $\nu^{2}=\sqrt{\tau}$ and implicit identification of $\alpha$ and $m_{q}$ as running quantities at this same scale. The running coupling has already been given in (51), and the running quark mass for three active flavours at two-loop order is

$$
m_{q}\left(\nu^{2}\right)=\frac{\hat{m}_{q}}{\left(\frac{1}{2} L\right)^{\frac{4}{9}}}\left(1+\frac{290}{729} \frac{1}{L}-\frac{256}{729} \frac{\log L}{L}\right) \quad, \quad L=\log \left(\frac{\nu^{2}}{\Lambda^{2}}\right),
$$

where $\hat{m}_{q}$ is the renormalization-group invariant quark mass parameter.

As for the vector channel, the QCD condensate contributions (61) do not have a branch discontinuity, so their contribution to the Gaussian sum-rule arises solely from the contour $\Gamma_{\epsilon}(\theta$ integral $)$ in (43), and can be evaluated using (46). 
The instanton contributions to the Gaussian sum-rule follow from (33)

$$
\begin{aligned}
G^{i n s t}(\hat{s}, \tau)= & (-1)^{I} \frac{3 m_{q}^{2}}{4 \pi^{2}} \frac{1}{\sqrt{4 \pi \tau}} \frac{1}{2 \pi i} \int_{\epsilon}^{\infty} t\left(\left[K_{1}\left(\rho_{c} \sqrt{t} e^{i \frac{\pi}{2}}\right)\right]^{2}-\left[K_{1}\left(\rho_{c} \sqrt{t} e^{-i \frac{\pi}{2}}\right)\right]^{2}\right) \exp \left(-\frac{(t-\hat{s})^{2}}{4 \tau}\right) d t \\
& +(-1)^{I} \frac{3 m_{q}^{2}}{4 \pi^{2}} \frac{1}{\sqrt{4 \pi \tau}} \frac{1}{2 \pi} \int_{-\pi}^{\pi} \epsilon^{2} e^{i 2 \theta}\left[K_{1}\left(\rho_{c} \sqrt{\epsilon} e^{i \frac{\theta}{2}}\right)\right]^{2} \exp \left(-\frac{\left(\epsilon e^{i \theta}+\hat{s}\right)^{2}}{4 \tau}\right) d \theta
\end{aligned}
$$

Simplification of (69) requires the following properties of the modified Bessel function $K_{1}(z)$ [25]

$$
\begin{aligned}
& K_{1}(z) \sim \frac{1}{z} \quad, \quad z \rightarrow 0 \\
& K_{1}(z)= \begin{cases}-\frac{\pi}{2} H_{1}^{(1)}\left(z e^{i \pi / 2}\right) \quad, \quad-\pi<\arg (z) \leq \frac{\pi}{2} \\
-\frac{\pi}{2} H_{1}^{(2)}\left(z e^{-i \pi / 2}\right) \quad, \quad-\frac{\pi}{2}<\arg (z) \leq \pi\end{cases}
\end{aligned}
$$

where $H_{1}^{(1)}(z)=J_{1}(z)+i Y_{1}(z)$ and $H_{1}^{(2)}(z)=J_{1}(z)-i Y_{1}(z)$. The asymptotic behaviour (70) implies that the $\theta$ integral of (69) will be zero in the $\epsilon \rightarrow 0$ limit and the identity (71) allows evaluation of the discontinuity in the $t$ integral of (69), leading to the following instanton contribution to the Gaussian sum-rule

$$
G^{\text {inst }}(\hat{s}, \tau)=-(-1)^{I} \frac{3 m_{q}^{2}}{8 \pi} \frac{1}{\sqrt{4 \pi \tau}} \int_{0}^{\infty} t J_{1}\left(\rho_{c} \sqrt{t}\right) Y_{1}\left(\rho_{c} \sqrt{t}\right) \exp \left(-\frac{(t-\hat{s})^{2}}{4 \tau}\right) d t
$$

Expressions (67), (46), (61), (43) and (72) lead to the Gaussian sum-rule of scalar currents

$$
\begin{aligned}
G(\hat{s}, \tau)= & \frac{1}{\sqrt{4 \pi \tau}} \frac{3 m_{q}^{2}(\sqrt{\tau})}{16 \pi^{2}} \int_{0}^{\infty}\left[t\left(1+\frac{17}{3} \frac{\alpha(\sqrt{\tau})}{\pi}\right)-2 \frac{\alpha(\sqrt{\tau})}{\pi} t \log \left(\frac{t}{\sqrt{\tau}}\right)\right] \exp \left(-\frac{(t-\hat{s})^{2}}{4 \tau}\right) d t \\
& -(-1)^{I} \frac{3 m_{q}^{2}}{8 \pi} \frac{1}{\sqrt{4 \pi \tau}} \int_{0}^{\infty} t J_{1}\left(\rho_{c} \sqrt{t}\right) Y_{1}\left(\rho_{c} \sqrt{t}\right) \exp \left(-\frac{(t-\hat{s})^{2}}{4 \tau}\right) d t \\
& +m_{q}^{2} \exp \left(-\frac{\hat{s}^{2}}{4 \tau}\right)\left[\frac{1}{2 \sqrt{\pi \tau}}\left\langle C_{4}^{s} \mathcal{O}_{4}^{s}\right\rangle-\frac{\hat{s}}{4 \tau \sqrt{\pi \tau}}\left\langle C_{6}^{s} \mathcal{O}_{6}^{s}\right\rangle\right] .
\end{aligned}
$$

Comparison of (72) and (8) indicates the existence of an instanton continuum contribution [26]

$$
\frac{1}{\pi} \operatorname{Im} \Pi^{\text {inst }}(t)=-(-1)^{I} \frac{3 m_{q}^{2}}{8 \pi} t J_{1}(\rho \sqrt{t}) Y_{1}(\rho \sqrt{t}) .
$$

Combined with the perturbative continuum devolving from $(60)$ we obtain the continuum contribution to the Gaussian sum-rule of scalar currents

$$
\begin{aligned}
G^{\text {cont }}(\hat{s}, \tau)= & \frac{1}{\sqrt{4 \pi \tau}} \frac{3 m_{q}^{2}(\sqrt{\tau})}{16 \pi^{2}} \int_{s_{0}}^{\infty}\left[t\left(1+\frac{17}{3} \frac{\alpha(\sqrt{\tau})}{\pi}\right)-2 \frac{\alpha(\sqrt{\tau})}{\pi} t \log \left(\frac{t}{\sqrt{\tau}}\right)\right] \exp \left(-\frac{(t-\hat{s})^{2}}{4 \tau}\right) d t \\
& -(-1)^{I} \frac{3 m_{q}^{2}}{8 \pi} \frac{1}{\sqrt{4 \pi \tau}} \int_{s_{0}}^{\infty} t J_{1}\left(\rho_{c} \sqrt{t}\right) Y_{1}\left(\rho_{c} \sqrt{t}\right) \exp \left(-\frac{(t-\hat{s})^{2}}{4 \tau}\right) d t
\end{aligned}
$$


and hence the theoretically determined Gaussian sum-rule (9) for the scalar currents

$$
\begin{aligned}
G^{Q C D}(\hat{s}, \tau)= & \frac{1}{\sqrt{4 \pi \tau}} \frac{3 m_{q}^{2}(\sqrt{\tau})}{16 \pi^{2}} \int_{0}^{s_{0}}\left[t\left(1+\frac{17}{3} \frac{\alpha(\sqrt{\tau})}{\pi}\right)-2 \frac{\alpha(\sqrt{\tau})}{\pi} t \log \left(\frac{t}{\sqrt{\tau}}\right)\right] \exp \left(-\frac{(t-\hat{s})^{2}}{4 \tau}\right) d t \\
& -(-1)^{I} \frac{3 m_{q}^{2}}{8 \pi} \frac{1}{\sqrt{4 \pi \tau}} \int_{0}^{s_{0}} t J_{1}\left(\rho_{c} \sqrt{t}\right) Y_{1}\left(\rho_{c} \sqrt{t}\right) \exp \left(-\frac{(t-\hat{s})^{2}}{4 \tau}\right) d t \\
& +m_{q}^{2} \exp \left(-\frac{\hat{s}^{2}}{4 \tau}\right)\left[\frac{1}{2 \sqrt{\pi \tau}}\left\langle C_{4}^{s} \mathcal{O}_{4}^{s}\right\rangle-\frac{\hat{s}}{4 \tau \sqrt{\pi \tau}}\left\langle C_{6}^{s} \mathcal{O}_{6}^{s}\right\rangle\right] .
\end{aligned}
$$

Finally, the quantity $M_{0}\left(\tau, s_{0}\right)$ in $(16)$ required for the normalized scalar-current Gaussian sum-rule (15) is given by

$$
\begin{aligned}
M_{0}\left(\tau, s_{0}\right)= & \frac{3 m_{q}^{2}(\sqrt{\tau})}{16 \pi^{2}}\left(1+\frac{17}{3} \frac{\alpha(\sqrt{\tau})}{\pi}\right) \frac{s_{0}^{2}}{2}-\frac{3 m_{q}^{2}(\sqrt{\tau})}{16 \pi^{2}} \frac{\alpha(\sqrt{\tau})}{\pi} \frac{s_{0}^{2}}{2}\left(2 \log \left(\frac{s_{0}}{\sqrt{\tau}}\right)-1\right) \\
& -(-1)^{I} \frac{3 m_{q}^{2}}{8 \pi} \int_{0}^{s_{0}} t J_{1}\left(\rho_{c} \sqrt{t}\right) Y_{1}\left(\rho_{c} \sqrt{t}\right) d t+m_{q}^{2}\left\langle C_{4}^{s} \mathcal{O}_{4}^{s}\right\rangle
\end{aligned}
$$

All theoretical contributions in (76) and (77) are proportional to $m_{q}^{2}$, and hence proportional to the $\hat{m}_{q}^{2}$ through (68). Consequently, the normalized Gaussian sum-rule $N^{Q C D}$ will be independent of $\hat{m}_{q}^{2}$ which is clearly advantageous given the uncertainty in determinations of the light-quark masses [10].

If the Gaussian sum-rules for scalar currents are analyzed using the single-resonance $\chi^{2}$-optimization techniques of Section 2.1 we find poor agreement between the theoretical prediction and the single resonance phenomenological model for the optimized values of the mass and continuum. Figures 6 and 5 show that the peak height of the single resonance model is consistently larger than the theoretical prediction, and that the peak position of the theoretical prediction shows some $\tau$ dependence. 6 Furthermore a discrepancy is seen at the tails of the distributions where the single-resonance model becomes smaller than the theoretical prediction. This latter point indicates that the width of the theoretical distribution is larger than that of the single resonance model.

A quantitative measure of this behaviour can be found in higher-order moments of the Gaussian sum-rule.

$$
\begin{aligned}
& M_{k}\left(\tau, s_{0}\right)=\int_{-\infty}^{\infty} \hat{s}^{k} G^{Q C D}\left(\hat{s}, \tau, s_{0}\right) d \hat{s} \quad, \quad k=0,1,2,3 \ldots \\
& \frac{M_{k}\left(\tau, s_{0}\right)}{M_{0}\left(\tau, s_{0}\right)}=\int_{-\infty}^{\infty} \hat{s}^{k} N^{Q C D}\left(\hat{s}, \tau, s_{0}\right) d \hat{s} \quad, \quad k=0,1,2,3 \ldots .
\end{aligned}
$$

Of particular importance are the moments related to the quantities $\sigma^{2}$ and $A_{2}$ defining the width and asymmetry of the distributions

$$
\begin{aligned}
\sigma^{2} & =\frac{M_{2}}{M_{0}}-\left(\frac{M_{1}}{M_{0}}\right)^{2} \\
A_{2} & =\frac{M_{3}}{M_{0}}-3 \frac{M_{2}}{M_{0}} \frac{M_{1}}{M_{0}}+2\left(\frac{M_{1}}{M_{0}}\right)^{3} .
\end{aligned}
$$

${ }^{6}$ We note that peak drift is absent in the equation $(23)$ expression for width effects, and furthermore unrealistically large resonance widths would be required to accommodate the observed discrepancy at the peak height. 
For the scalar channel, the relevant higher-order moments for our analysis are given by

$$
\begin{aligned}
M_{1}\left(\tau, s_{0}\right)= & \frac{3 m_{q}^{2}(\sqrt{\tau})}{16 \pi^{2}}\left(1+\frac{17}{3} \frac{\alpha(\sqrt{\tau})}{\pi}\right) \frac{s_{0}^{3}}{3}-\frac{3 m_{q}^{2}(\sqrt{\tau})}{16 \pi^{2}} \frac{\alpha(\sqrt{\tau})}{\pi} \frac{2 s_{0}^{3}}{9}\left(3 \log \left(\frac{s_{0}}{\sqrt{\tau}}\right)-1\right) \\
& -(-1)^{I} \frac{3 m_{q}^{2}}{8 \pi} \int_{0}^{s_{0}} t^{2} J_{1}\left(\rho_{c} \sqrt{t}\right) Y_{1}\left(\rho_{c} \sqrt{t}\right) d t-m_{q}^{2}\left\langle C_{6}^{s} \mathcal{O}_{6}^{s}\right\rangle \\
M_{2}\left(\tau, s_{0}\right)= & \frac{3 m_{q}^{2}(\sqrt{\tau})}{16 \pi^{2}}\left(1+\frac{17}{3} \frac{\alpha(\sqrt{\tau})}{\pi}\right)\left[\frac{s_{0}^{4}}{4}+\tau s_{0}^{2}\right] \\
& -\frac{3 m_{q}^{2}(\sqrt{\tau})}{16 \pi^{2}} \frac{\alpha(\sqrt{\tau})}{\pi} \frac{s_{0}^{2}}{8}\left[s_{0}^{2}\left(4 \log \left(\frac{s_{0}}{\sqrt{\tau}}\right)-1\right)+8 \tau\left(2 \log \left(\frac{s_{0}}{\sqrt{\tau}}\right)-1\right)\right] \\
& -(-1)^{I} \frac{3 m_{q}^{2}}{8 \pi} \int_{0}^{s_{0}} t\left(t^{2}+2 \tau\right) J_{1}\left(\rho_{c} \sqrt{t}\right) Y_{1}\left(\rho_{c} \sqrt{t}\right) d t+m_{q}^{2} 2 \tau\left\langle C_{4}^{s} \mathcal{O}_{4}^{s}\right\rangle \\
M_{3}\left(\tau, s_{0}\right)= & \frac{3 m_{q}^{2}(\sqrt{\tau})}{16 \pi^{2}}\left(1+\frac{17}{3} \frac{\alpha(\sqrt{\tau})}{\pi}\right)\left[\frac{s_{0}^{5}}{5}+2 \tau s_{0}^{3}\right] \\
& -\frac{3 m_{q}^{2}(\sqrt{\tau})}{16 \pi^{2}} \frac{\alpha(\sqrt{\tau})}{\pi} 2 s_{0}^{3}\left[\frac{s_{0}^{2}}{25}\left(5 \log \left(\frac{s_{0}}{\sqrt{\tau}}\right)-1\right)+\frac{2}{3} \tau\left(3 \log \left(\frac{s_{0}}{\sqrt{\tau}}\right)-1\right)\right] \\
& -(-1)^{I} \frac{3 m_{q}^{2}}{8 \pi} \int_{0}^{s_{0}} t^{2}\left(t^{2}+6 \tau\right) J_{1}\left(\rho_{c} \sqrt{t}\right) Y_{1}\left(\rho_{c} \sqrt{t}\right) d t-m_{q}^{2} 6 \tau\left\langle C_{6}^{s} \mathcal{O}_{6}^{s}\right\rangle
\end{aligned}
$$

For completeness, we also give the moments needed to calculate $\sigma^{2}$ for the vector channel.

$$
\begin{aligned}
& M_{1}\left(\tau, s_{0}\right)=\frac{1}{8 \pi^{2}}\left(1+\frac{\alpha(\sqrt{\tau})}{\pi}\right) \frac{s_{0}^{2}}{2}-\frac{1}{8 \pi^{2}}\left\langle C_{4}^{v} \mathcal{O}_{4}^{v}\right\rangle \\
& M_{2}\left(\tau, s_{0}\right)=\frac{1}{8 \pi^{2}}\left(1+\frac{\alpha(\sqrt{\tau})}{\pi}\right) s_{0}\left(\frac{1}{3} s_{0}^{2}+2 \tau\right)+\frac{1}{8 \pi^{2}}\left\langle C_{6}^{v} \mathcal{O}_{6}^{v}\right\rangle
\end{aligned}
$$

Consider a two-resonance extension of the narrow resonance model (11) so that (12) becomes

$$
G^{Q C D}\left(\hat{s}, \tau, s_{0}\right)=\frac{f_{1}^{2}}{\sqrt{4 \pi \tau}} \exp \left[-\frac{\left(\hat{s}-m_{1}^{2}\right)^{2}}{4 \tau}\right]+\frac{f_{2}^{2}}{\sqrt{4 \pi \tau}} \exp \left[-\frac{\left(\hat{s}-m_{2}^{2}\right)^{2}}{4 \tau}\right]
$$

The corresponding expression for the normalized Gaussian sum-rule then becomes

$$
N^{Q C D}\left(\hat{s}, \tau, s_{0}\right)=\frac{1}{\sqrt{4 \pi \tau}}\left\{r_{1} \exp \left[-\frac{\left(\hat{s}-m_{1}^{2}\right)^{2}}{4 \tau}\right]+r_{2} \exp \left[-\frac{\left(\hat{s}-m_{2}^{2}\right)^{2}}{4 \tau}\right]\right\}
$$

where

$$
r_{1}=\frac{f_{1}^{2}}{f_{1}^{2}+f_{2}^{2}} \quad, \quad r_{2}=\frac{f_{2}^{2}}{f_{1}^{2}+f_{2}^{2}} \quad, \quad r_{1}+r_{2}=1
$$

If we calculate moments of (87), we find that in the two-resonance model

$$
\begin{aligned}
& \frac{M_{1}}{M_{0}}=r_{1} m_{1}^{2}+r_{2} m_{2}^{2}=\frac{1}{2}(z+r y) \\
& \sigma^{2}-2 \tau=r_{1} r_{2}\left(m_{1}^{2}-m_{2}^{2}\right)^{2}=\frac{1}{4} y^{2}\left(1-r^{2}\right) \\
& A_{2}=r_{1} r_{2}\left(r_{2}-r_{1}\right)\left(m_{1}^{2}-m_{2}^{2}\right)^{3}=-\frac{1}{4} r y^{3}\left(1-r^{2}\right)
\end{aligned}
$$


where

$$
\begin{aligned}
& r=r_{1}-r_{2} \\
& y=m_{1}^{2}-m_{2}^{2} \\
& z=m_{1}^{2}+m_{2}^{2} .
\end{aligned}
$$

We see that in the single resonance limit, $\sigma^{2}=2 \tau$ and $A_{2}=0$, and hence a clear signature of the existence of two resonances is $\sigma^{2}-2 \tau \neq 0$. ฤ

In Figures 6 and $7, \sigma^{2}$ is plotted as a function of $\tau$ for the optimum values of $s_{0}$ resulting from the singleresonance $\chi^{2}$ analyses of the $I=0,1$ scalar currents. From Figures 6 and 7 it is evident that $\sigma^{2}-2 \tau$ is significantly different from zero, indicating the presence of a second resonance in both the $I=0,1$ scalar channels. In contrast, Figure 8 for the vector channel shows that $\sigma^{2}$ differs insignificantly from $2 \tau$. Consequently, there is no evidence that a second vector resonance has enough strength to necessitate extension of the phenomenological model, implying that any such states are weak enough to be absorbed into the continuum, and providing a validation of the singleresonance analysis. Compared to the Laplace sum-rules which exponentially suppress excited states, the potential sensitivity to excited states is a unique feature of the Gaussian sum-rules.

How might we extract predictions of the hadronic parameters $\left\{m_{1}, r_{1}, m_{2}, r_{2}\right\}$ (or equivalently $\{r, y, z\}$ ) from the Gaussian sum-rules (10) in a two narrow resonance model? The key to solving this problem lies in the behaviour of the peak position $\hat{s}_{\text {peak }}\left(\tau, s_{0}\right)$ of $N^{\mathrm{QCD}}\left(\hat{s}, \tau, s_{0}\right)$. As noted in Section 2.1, in the single narrow resonance model, the phenomenological side of (17) possesses a $\tau$-independent peak located at $\hat{s}=m_{r}^{2}$. In the double narrow resonance model (88), the situation is more complicated. For any fixed value of $\tau$ sufficiently large compared to $m_{2}^{2}-m_{1}^{2}$ (in practice, this is indeed the case), the right-hand-side of (88) exhibits a single peak; however, the position of this peak varies with $\tau$, and it is this $\tau$-dependent peak drift which enables us to extract hadronic parameters in the double narrow resonance model.

To determine an expression for the phenomenological peak drift, we find the location of the peak by differentiating the right-hand-side of (88) and setting the result equal to zero. In terms of the parameters (93 95), this yields

$$
\frac{(r+1)\left(\hat{s}-\frac{1}{2} z-\frac{1}{2} y\right)}{(r-1)\left(\hat{s}-\frac{1}{2} z+\frac{1}{2} y\right)}-\exp \left[\frac{y(z-2 \hat{s})}{4 \tau}\right]=0 .
$$

Unfortunately, we cannot explicitly solve (96) for $\hat{s}$ as a function of $\tau$, so we instead compute the peak drift as a series expansion in the parameter $1 / \tau$ :

$$
A+\frac{B}{\tau}+\frac{C}{\tau^{2}}
$$

where $A, B$, and $C$ are functions of the parameters $\{r, y, z\}$, and the quantities $\{B, C\}$ will be non-zero except in the single resonance limit $(y=0)$ or in the case of equal resonance strengths $(r=0)$. Explicit numerical testing in worst case scenarios involving two resonances with masses in the $1-2 \mathrm{GeV}$ range show that the first omitted term in (97) [i.e. $D / \tau^{3}$ ] is negligible provided that we restrict our attention to $\tau \geq 2 \mathrm{GeV}^{4}$.

We define the following $\chi^{2}$ function which measures quantitatively the discrepency between the theoretical peak drift contained in $N^{\mathrm{QCD}}\left(\hat{s}, \tau, s_{0}\right)$ and the phenomenological peak drift approximated by (97)

$$
\chi^{2}\left(s_{0}\right)=\sum_{i=1}^{N}\left[\hat{s}_{\text {peak }}\left(s_{0}, \tau_{i}\right)-A\left(s_{0}\right)-\frac{B\left(s_{0}\right)}{\tau_{i}}-\frac{C\left(s_{0}\right)}{\tau_{i}^{2}}\right]^{2},
$$

where $A\left(s_{0}\right), B\left(s_{0}\right)$, and $C\left(s_{0}\right)$ are obtained by solving the linear system of equations defined by the $\chi^{2}$-minimizing conditions

$$
\frac{\partial \chi^{2}}{\partial A}=\frac{\partial \chi^{2}}{\partial B}=\frac{\partial \chi^{2}}{\partial C}=0
$$

\footnotetext{
${ }^{7}$ Note that $A_{2}=0$ for resonances of equal strength $r_{1}=r_{2}$, and hence $A_{2}=0$ does not necessarily imply a single resonance scenario.
} 
Minimizing (98) numerically using the Golden search algorithm [5] determines an optimum value for $s_{0}$. At this optimum $s_{0}$, the moments (78) exhibit negligible dependence on $\tau$ as explicitly occurs in the double resonance model, and thus the moments are well approximated on the entire $\tau$ region of interest by averaged values

$$
\overline{M_{i}\left(s_{0}\right)}=\frac{1}{\tau_{f}-\tau_{i}} \int_{\tau_{i}}^{\tau_{f}} M_{i}\left(\tau, s_{0}\right) d \tau .
$$

Next, we calculate $\overline{\sigma^{2}-2 \tau}$ and $\overline{A_{2}}$ by substituting the average moments 100 into (79) and (80). Finally, we substitute these quantities along with $\overline{M_{0}}$ and $\overline{M_{1}}$ into the following inversion formulae which follow easily from 90 92):

$$
\begin{aligned}
& z=2 \frac{M_{1}}{M_{0}}+\frac{A_{2}}{\sigma^{2}-2 \tau} \\
& y=\frac{-\sqrt{A_{2}^{2}+4\left(\sigma^{2}-2 \tau\right)^{3}}}{\sigma^{2}-2 \tau} \\
& r=\frac{A_{2}}{\sqrt{A_{2}^{2}+4\left(\sigma^{2}-2 \tau\right)^{3}}}
\end{aligned}
$$

to arrive at our predictions for the hadronic parameters $\{r, y, z\}$ in the double narrow resonance model.

These techniques for analyzing the Gaussian sum-rule for a two-resonance phenomenological model can now be applied to the $\bar{n} n$ quark scalar mesons. The range $2.0 \mathrm{GeV}^{4} \leq \tau \leq 4.0 \mathrm{GeV}^{4}$ will be used to define the $\chi^{2}$ (98) since it leads to acceptable convergence of the series solution (97) for the peak drift as well as minimizing uncertainties from the (large) higher order perturbative coefficients in (76). Table 1 shows the Gaussian sum-rule prediction of the quark scalar resonance parameters and continuum resulting from the two-resonance $\chi^{2}$-optimization procedure. Figures 9 and 10 compare the $\hat{s}$ dependence of the Gaussian sum-rule and the two-resonance model with the Table 1 predictions of the resonance parameters. The nearly-perfect agreement between the theoretical prediction and two-resonance phenomenological model exhibited in Figures 9 and 10 is clearly a vast improvement compared with the one-resonance model results displayed in Figures 4 and 5, and provides compelling evidence for the ability of Gaussian sum-rules to predict resonance properties. The absence of any indication of disagreement between the theoretical prediction and two-resonance model suggests that any further resonances are weak enough to be contained in the QCD continuum.

The prediction of the resonance parameters for the $\bar{n} n$ scalar resonances are of phenomenological interest. The $I=0$ states in Table 1 suggest that the $f_{0}(980)$ has a significant $\bar{n} n$ component, with a weaker excited state with an $\bar{n} n$ component lying in the vicinity of the $f_{0}(1370)$ and $f_{0}(1500)$. The absence of a light (substantially less than $1 \mathrm{GeV}) I=0$ state indicates a decoupling of a light $\sigma$ from the $\bar{n} n$ scalar currents, suggesting a non- $\bar{n} n$ interpretation for the $\sigma$. The $I=1$ states in Table 1 similarly suggest a non- $\bar{n} n$ interpretation of the $a_{0}(980)$, and are consistent with identification of the $a_{0}(1450)$ as the lightest $I=1$ state with a significant $\bar{n} n$ content. Our prediction of an $I=1$ state near $1.8 \mathrm{GeV}$ suggests identification of the the $I=1$ state $X(1775)$ 10 as a scalar state. Finally, we note that the Gaussian sum-rule predictions for the lightest $I=0,1 \bar{n} n$ scalar mesons confirm the results obtained from Laplace sum-rules [27] which, because of the their exponential suppression of excited states, are best suited to probing the ground states in these channels.

\begin{tabular}{||c|c|c|c|c|c||}
\hline \hline$I$ & $m_{1}$ & $m_{2}$ & $r_{1}$ & $r_{2}$ & $s_{0}$ \\
\hline \hline 0 & $0.97 \mathrm{GeV}$ & $1.43 \mathrm{GeV}$ & 0.634 & 0.366 & $2.35 \mathrm{GeV}^{2}$ \\
\hline 1 & $1.44 \mathrm{GeV}$ & $1.81 \mathrm{GeV}$ & 0.570 & 0.430 & $3.50 \mathrm{GeV}^{2}$ \\
\hline \hline
\end{tabular}

Table 1: Results of the two-resonance analysis of the Gaussian sum-rules for the $I=0,1 \bar{n} n$ quark scalar mesons resulting from the $\chi^{2}$-optimization procedure. Central values of the QCD parameters have been employed.

As a check on the methods developed for analyzing the two-resonance case, we have also performed a leastsquares fit between the complete two-dimensional $(\hat{s}, \tau)$ dependence of the two-resonance phenomenological model and the theoretical prediction. The resonance parameters and continuum parameters obtained from this analysis 
differ from those of Table 1 by at most 10\%, demonstrating that the peak-drift method reproduces a full fitting procedure with minimal computational expense, and provides unambiguous results compared to a full multi-dimensional least-squares fit. At the very least, the peak-drift method can be used to initialize a full multi-dimensional leastsquares fitting procedure.

\section{Conclusions}

In this paper we have developed techniques for using QCD Gaussian sum-rules to predict hadronic resonance properties, and discussed the conceptual advantages of the Gaussian sum-rule in providing a natural duality interval for the relation between QCD and hadronic physics. Methods for analyzing a single resonance model based on the $\hat{s}$ peak position and peak height of the theoretical prediction for the Gaussian sum-rule were developed and applied to the $\rho$ meson as a test case. Motivated by the accuracy of the $\rho$ mass prediction and exceptional agreement between the theoretical prediction and single resonance phenomenological model (see Figure 3), the more phenomenologically challenging case of the non-strange $\bar{n} n$ quark scalar mesons was considered. However, the single resonance analysis of the Gaussian sum-rule of the quark scalar currents revealed a discrepancy between the theoretical prediction and phenomenological model (see Figures 4 and 5).

This discrepancy between the theoretical prediction and single resonance model for the scalar currents is addressed by including a second resonance in the phenomenological model. The quantity $\sigma^{2}$, defined in (80) by moments of the Gaussian sum-rule, provides a criterion for evaluating the necessity of including a second resonance in the phenomenological model. Figures 6 8 illustrate that the single resonance model is validated for the vector case, but is insufficient for the scalar currents. Compared with the Laplace sum-rule, the potential sensitivity of the Gaussian sum-rule to excited states is a novel feature of the Gaussian sum-rules.

The methods developed for analyzing the Gaussian sum-rules in a single resonance phenomenological model can be extended to the two-resonance model by studying the $\tau$ dependence of the $\hat{s}$ peak position (peak drift) of the theoretical prediction for the Gaussian sum-rule. When applied to the Gaussian sum-rule of $\bar{n} n$ quark scalar currents, we find the discrepancy between the theoretical prediction and phenomenological model observed in the single-resonance analysis is resolved by inclusion of a second resonance, and the resulting agreement between the theoretical prediction and phenomenological model (see Figures 9 and 10) is astounding.

The phenomenological results for the $\bar{n} n$ quark scalar mesons (see Table 1) provide valuable information for interpretation of the scalar mesons. In particular, the Gaussian sum-rule analysis is in excellent agreement with the identification of the $a_{0}(1450)$ as the lightest quark scalar meson with a significant $\bar{n} n$ content, supporting the conclusions of [27, 28], and suggests that a light $(\ll 1 \mathrm{GeV}) I=0$ scalar meson does not have a significant $\bar{n} n$ component. The $I=1$ excited state in Table 1 suggests identification of the $X(1775)$ [10] as a scalar state containing $\bar{n} n$.

In summary, we conclude that the Gaussian sum-rules are not only useful in their well-established connection with the finite-energy sum-rule constraint on the continuum threshold [3], but are a valuable technique for studying hadronic physics, and should be considered as a viable and complementary alternative to Laplace sum-rules.

Acknowledgements: TGS is grateful for the warm hospitality of GO and W. Leidemann at the Università di Trento while this research was conducted. TGS and DH also acknowledge research support from the Natural Sciences and Engineering Research Council of Canada (NSERC).

\section{References}

[1] M.A. Shifman, A.I. Vainshtein and V.I. Zakharov, Nucl. Phys. B147 (1979) 385, 448;

L.J. Reinders, H. Rubenstein and S. Yazaki, Phys. Rep. 1 (1985) 1.

[2] R. Shankar, Phys. Rev. D15 (1977) 755;

R.G. Moorhouse, M.R. Pennington, G.G. Ross, Nucl. Phys. B214 (1977) 285;

K.G. Chetyrkin, N.V. Krasnikov, N.N. Tavkhelidze Phys. Lett. 76B (1978) 83;

E.G. Floratos, S. Narison, E. de Rafael, Nucl. Phys. B155 (1979) 115. 
[3] R.A. Bertlmann, G. Launer, E. de Rafael, Nucl. Phys. B250 (1985) 61.

[4] V.D. Efros, W. Leidemann, G. Orlandini, Phys. Rev. Lett. 78 (1997) 432, 4015.

[5] W.K. Press et. al., Numerical Recipes in C: the Art of Scientific Computing, (Cambridge University Press, Cambridge, 1992).

[6] V. Elias, A.H. Fariborz, M.A. Samuel, Fang Shi, T.G. Steele, Phys. Lett. B412 (1997) 131;

V. Elias, A.H. Fariborz, Fang Shi, T.G. Steele, Nucl. Phys. A633 (1998) 279.

[7] S.G. Gorishny, A.L. Kataev, S.A. Larin, Phys. Lett. B259 (1991) 144.

[8] V. Giménez, J. Bordes, J. Peñarrocha, Phys. Lett. B223 (1989) 251;

S.N. Nikolaev, H.R. Radyushkin, Nucl. Phys. B213 (1983) 285;

D.J. Broadhurst, S.C. Generalis, Phys. Lett. B165 (1985) 175.

[9] V. Giménez, J. Bordes, J. Peñarrocha, Nucl. Phys. B357 (1991) 3.

[10] C. Caso et al, Eur. Phys. J. C3 (1998) 1.

[11] T.G. Steele and V. Elias, Mod. Phys. Lett. A13 (1998) 3151.

[12] K.G. Chetyrkin, B.A. Kneihl and M. Steinhauser, Nucl. Phys. B510 (1998) 61.

[13] M. Gell-Mann, R.J. Oakes, B. Renner, Phys. Rev. 175 (1968) 2195.

[14] C.A. Dominguez, J. Sola, Z. Phys. C40 (1988) 63.

[15] Y. Nambu, G. Jona-Lasinio, Phys. Rev. 122 (1961) 345;

V. Elias, M.D. Scadron, Phys. Rev. Lett. 53 (1984) 1129;

V.A. Miransky, M.D. Scadron, Sov. J. Nucl. Phys. 49 (1989) 922;

J. Weinstein, N. Isgur, Phys. Rev. D41 (1990) 2236;

D. Morgan, M.R. Pennington, Phys. Rev. D48 (1993) 1185;

N.A. Törnqvist, Z. Phys. C68 (1995) 647;

S. Narison, Nucl. Phys. B509 (1998) 312;

P. Minkowski, W. Ochs, Eur. Phys. J. C9 (1999) 283;

J.A. Oller, E. Oset, Nucl. Phys. A663-664 (2000) 629.

[16] G. Jannsen, B.C. Pearce, K. Holinde, J. Speth, Phys. Rev. D52 (1995) 2690.

[17] R.L. Jaffe, Phys. Rev. D15 (1977) 267;

D. Black, A.H. Fariborz, F. Sannino, J. Schechter, Phys. Rev. D59 (1999) 074026.

[18] D.G. Caldi: Phys. Rev. Lett. 39 (1977) 121;

C.G. Callan, R. Dashen, and D. Gross: Phys. Rev. D16 (1977) 2526 and D17 (1978) 2717;

V.A. Novikov, M.A. Shifman, A.I. Vainshtein, and V.I. Zakharov: Nucl. Phys. B191 (1981) 301;

E. Gabrielli and P. Nason: Phys. Lett. B313 (1993) 430.

[19] E.V. Shuryak: Nucl. Phys. B214 (1983) 237.

[20] R.D. Carlitz: Phys. Rev. D17 (1978) 3225;

R.D. Carlitz and D.B. Creamer: Ann. Phys. 118 (1979) 429.

[21] K.G. Chetyrkin, Phys. Lett. B390 (1997) 309;

S.G. Gorishny, A.L. Kataev, S.A. Larin, L.R. Surguladze, Phys. Rev. D43 (1991) 1633 and Mod. Phys. Lett. A5 (1990) 2703.

[22] E. Bagan, J.I. LaTorre, and P. Pascual, Z. Phys. C32 (1986) 43. 
[23] L.J. Reinders, S. Yazaki, and H.R. Rubinstein, Nucl. Phys. B196 (1982) 125.

[24] E.V. Shuryak, Nucl. Phys. B214 (1983) 237.

[25] M. Abramowitz and I.E. Stegun, Mathematical Functions with Formulas, Graphs, and Mathematical Tables (National Bureau of Standards Applied Mathematics Series, Washington) 1972.

[26] V. Elias, Fang Shi, T.G. Steele, J. Phys. G24 (1998) 267;

A.S. Deakin, V. Elias, Ying Xue, N.H. Fuchs, Fang Shi, T.G. Steele, Phys. Lett. B418 (1998) 223.

[27] Fang Shi, T.G. Steele, V. Elias, K.B. Sprague, Ying Xue, A.H. Fariborz, Nucl. Phys. A671 (2000) 416.

[28] L. Burakovsky, T. Goldman, Nucl. Phys. A628 (1998) 87;

D. Black, A.H. Fariborz, J. Schechter, Phys. Rev. D61 (2000) 074001;

J.A. Oller and E. Oset, Phys. Rev. D60 (1999) 074023;

M. Jamin, J.A. Oller and A. Pich, hep-ph/0006045. 


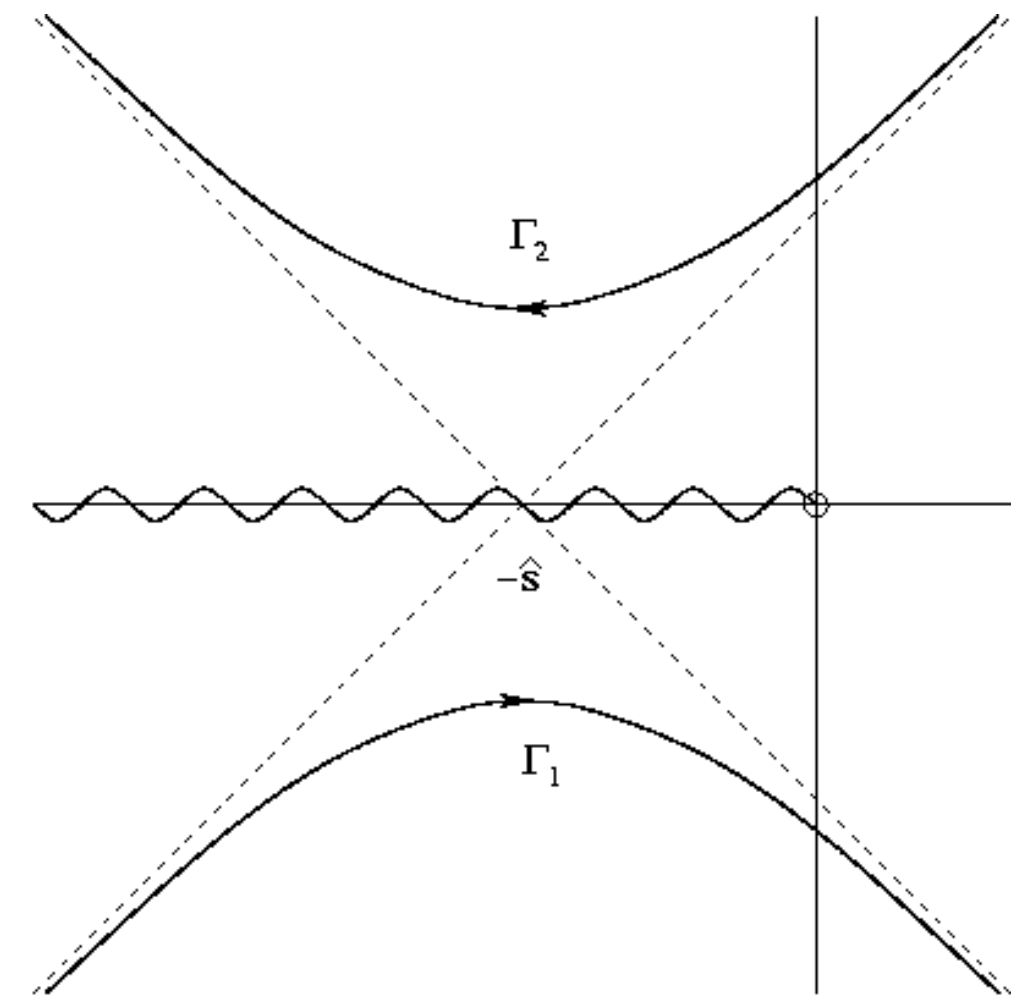

Figure 1: Contour of integration $\Gamma_{1}+\Gamma_{2}$ defining the Gaussian sum-rule in (34). The wavy line on the negative real axis denotes the branch cut of $\Pi(w)$. 


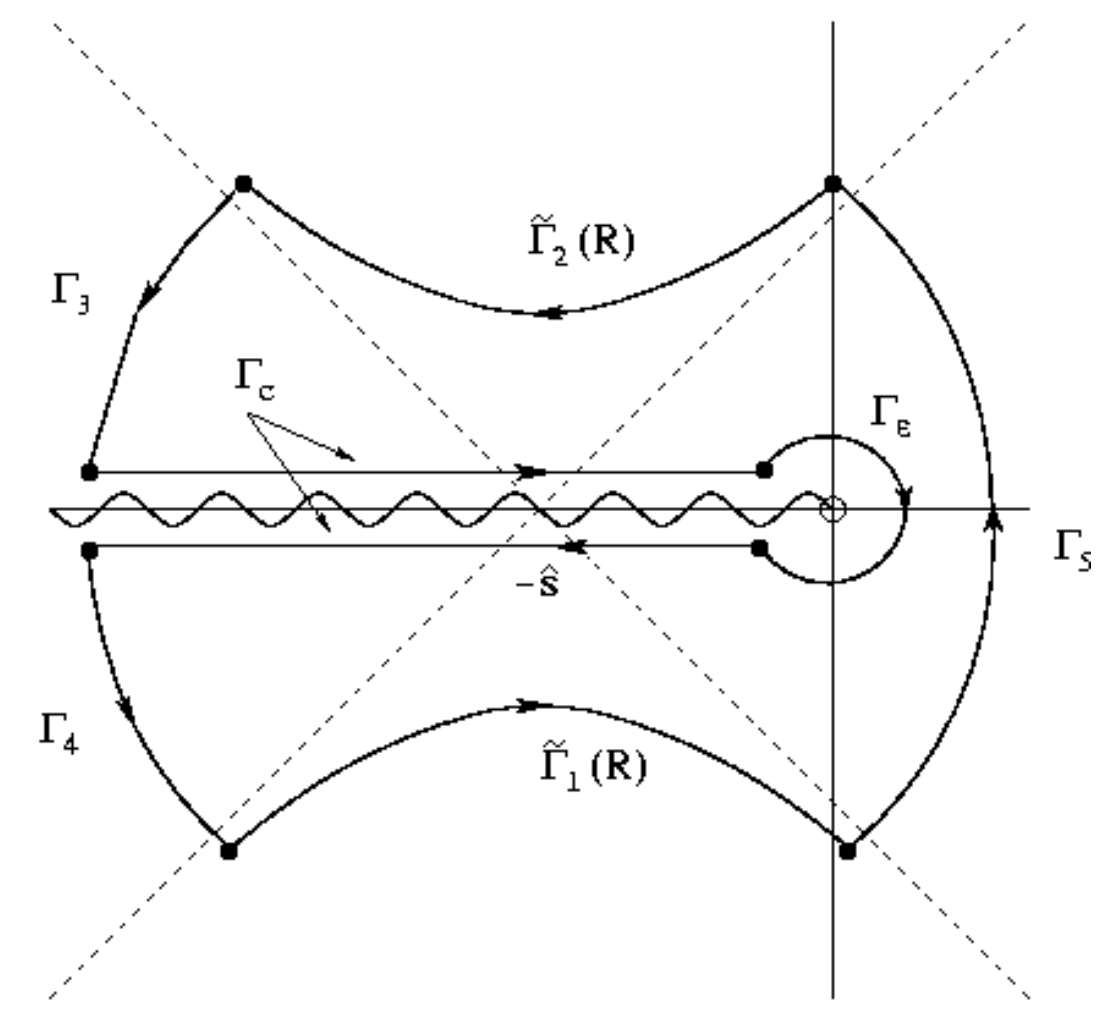

Figure 2: Closed contour $C(R)$ used to calculate the Gaussian sum-rule defined by (34). The inner circular segment $\Gamma_{\epsilon}$ has a radius of $\epsilon$, and the circular segments $\Gamma_{3}, \Gamma_{4}$ and $\Gamma_{5}$ have a radius $R$. The wavy line on the negative real axis denotes the branch cut of $\Pi(w)$, and the linear segments of the contour above and below the branch cut are denoted by $\Gamma_{c}$. The contour $\tilde{\Gamma}_{1}(R)$ is that portion of $\Gamma_{1}$ (see Figure 1 ) which lies in the interior of a circle of radius $R$ centred at $-\hat{s}$, and similarly for $\tilde{\Gamma}_{2}(R)$. 


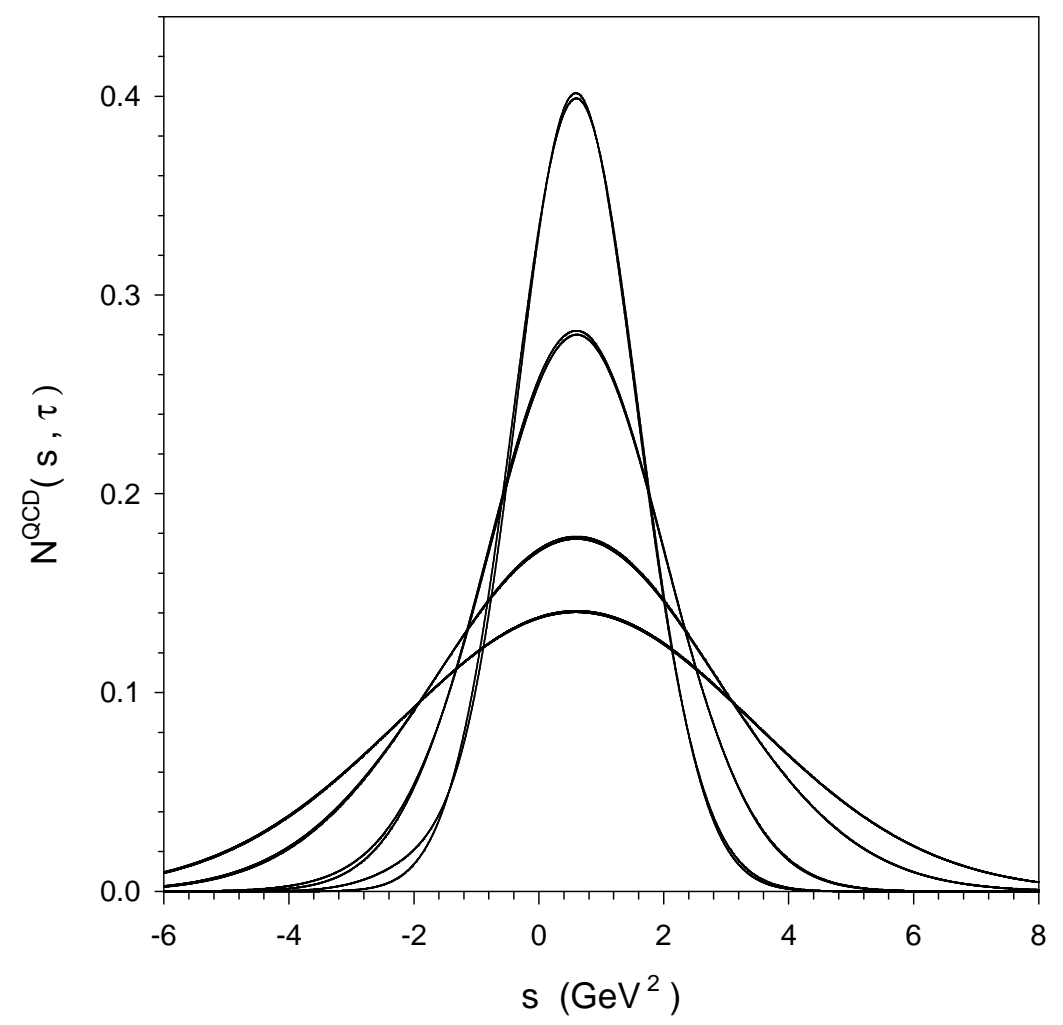

Figure 3: Comparison of the vector-current theoretical prediction for the $N^{Q C D}\left(\hat{s}, \tau, s_{0}\right)$ with the single resonance phenomenological model using the $\chi^{2}$-optimized values of the resonance mass $m_{\rho}=0.773 \mathrm{GeV}$ and continuum $s_{0}=1.22 \mathrm{GeV}^{2}$. The $\tau$ values used for the four pairs of curves, from top to bottom in the figure, are respectively $\tau=0.5 \mathrm{GeV}^{4}, \tau=1.0 \mathrm{GeV}^{4}, \tau=2.5 \mathrm{GeV}^{4}$, and $\tau=4.0 \mathrm{GeV}^{4}$. Note the almost perfect overlap between the theoretical prediction and phenomenological model. Central values of the condensate parameters have been used. 


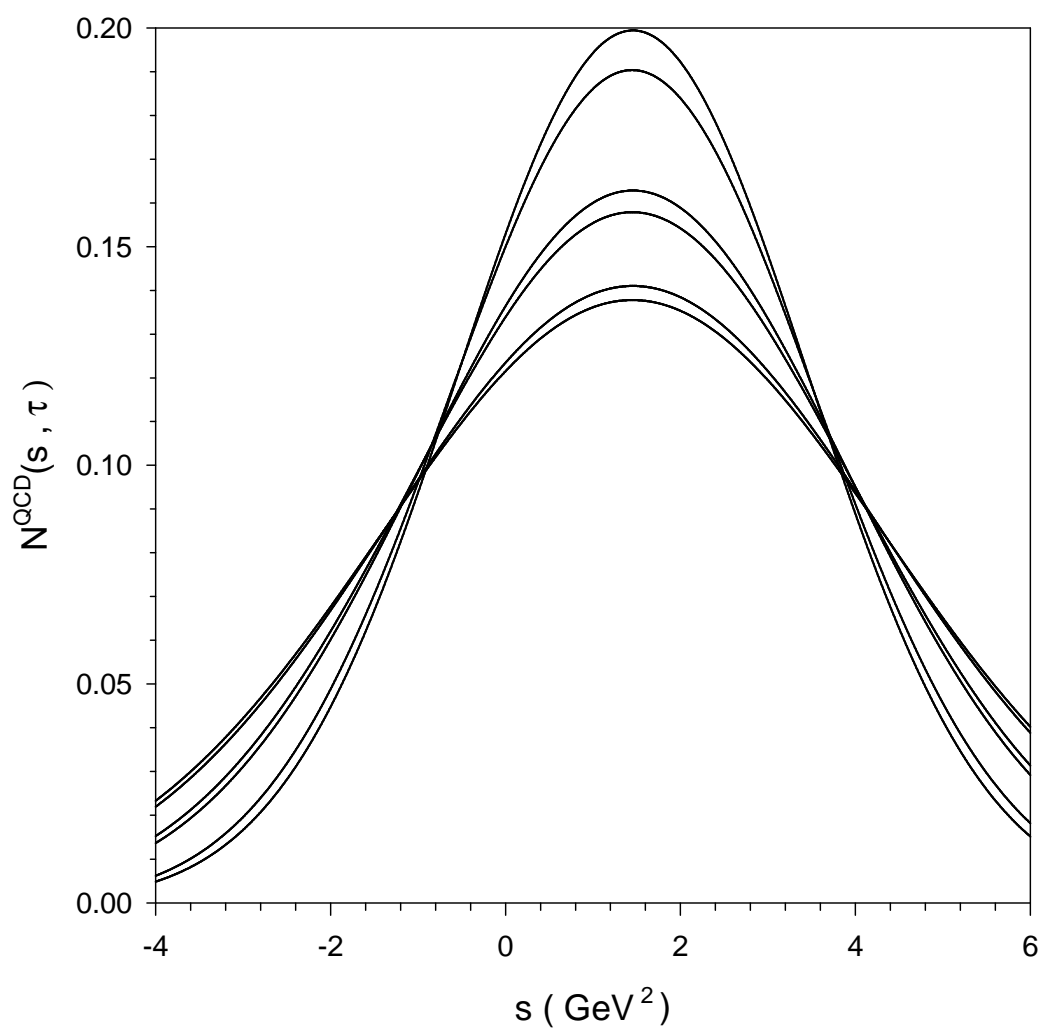

Figure 4: Comparison of the $I=0$ scalar current theoretical prediction for $N^{Q C D}\left(\hat{s}, \tau, s_{0}\right)$ with the single resonance phenomenological model using the $\chi^{2}$-optimized values of the resonance mass $m=1.21 \mathrm{GeV}$ and continuum $s_{0}=$ $2.60 \mathrm{GeV}^{2}$. The $\tau$ values used for the three pairs of curves, from top to bottom in the figure, are respectively $\tau=2.0 \mathrm{GeV}^{4}, \tau=3.0 \mathrm{GeV}^{4}$, and $\tau=4.0 \mathrm{GeV}^{4}$. The phenomenological model is consistently larger than the theoretical prediction near the peak, but is consistently smaller than the theoretical prediction in the tails. Central values of the condensate parameters have been used. 


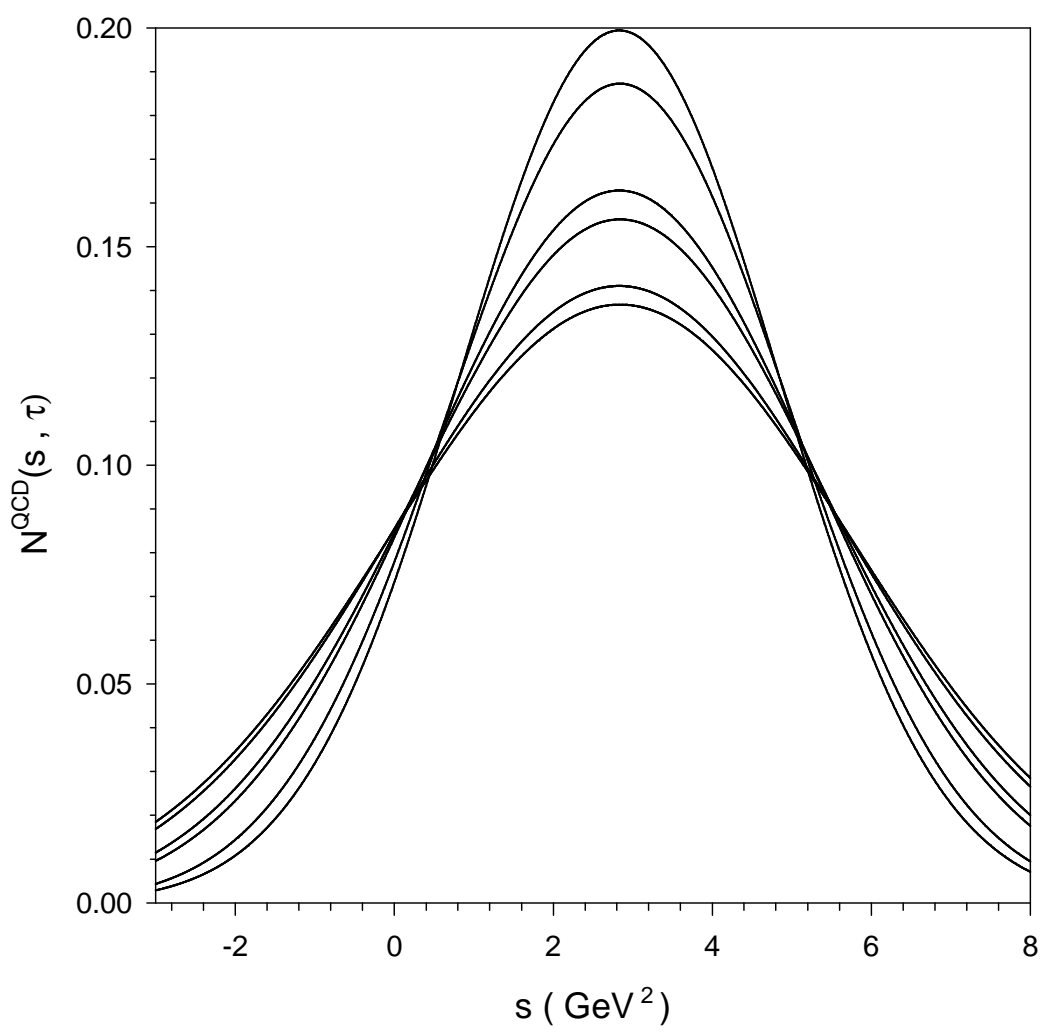

Figure 5: Comparison of the $I=1$ scalar current theoretical prediction for $N^{Q C D}\left(\hat{s}, \tau, s_{0}\right)$ with the single resonance phenomenological model using the $\chi^{2}$-optimized values of the resonance mass $m=1.68 \mathrm{GeV}$ and continuum $s_{0}=$ $3.9 \mathrm{GeV}^{2}$. The $\tau$ values used for the three pairs of curves, from top to bottom in the figure, are respectively $\tau=2.0 \mathrm{GeV}^{4}, \tau=3.0 \mathrm{GeV}^{4}$, and $\tau=4.0 \mathrm{GeV}^{4}$. The phenomenological model is consistently larger than the theoretical prediction near the peak, but is consistently smaller than the theoretical prediction in the tails. Central values of the condensate parameters have been used. 


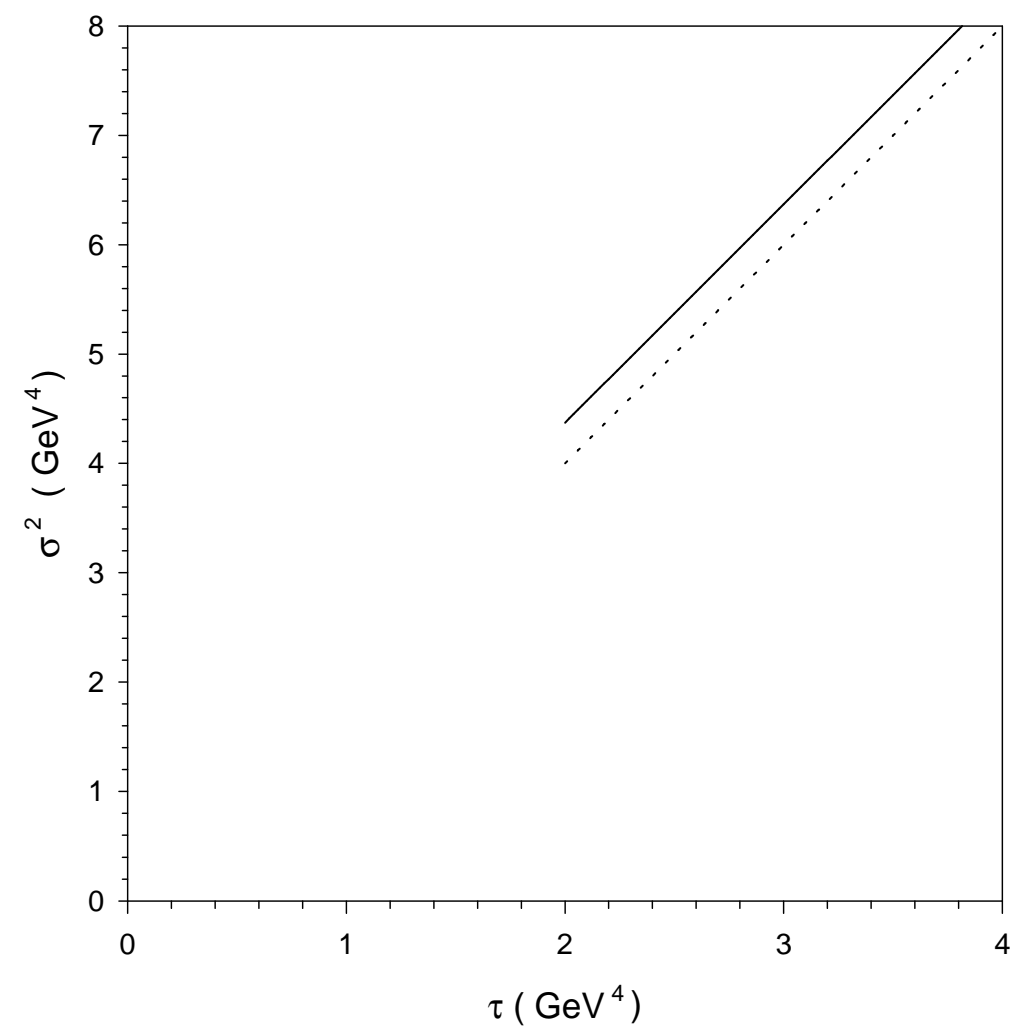

Figure 6: Plot of $\sigma^{2}$ for the theoretical prediction (solid curve) compared with $\sigma^{2}=2 \tau$ for the single-resonance model (dashed curve) for the $I=0$ scalar channel using the $\chi^{2}$-optimized value of the continuum $s_{0}=2.6 \mathrm{GeV}^{2}$. 


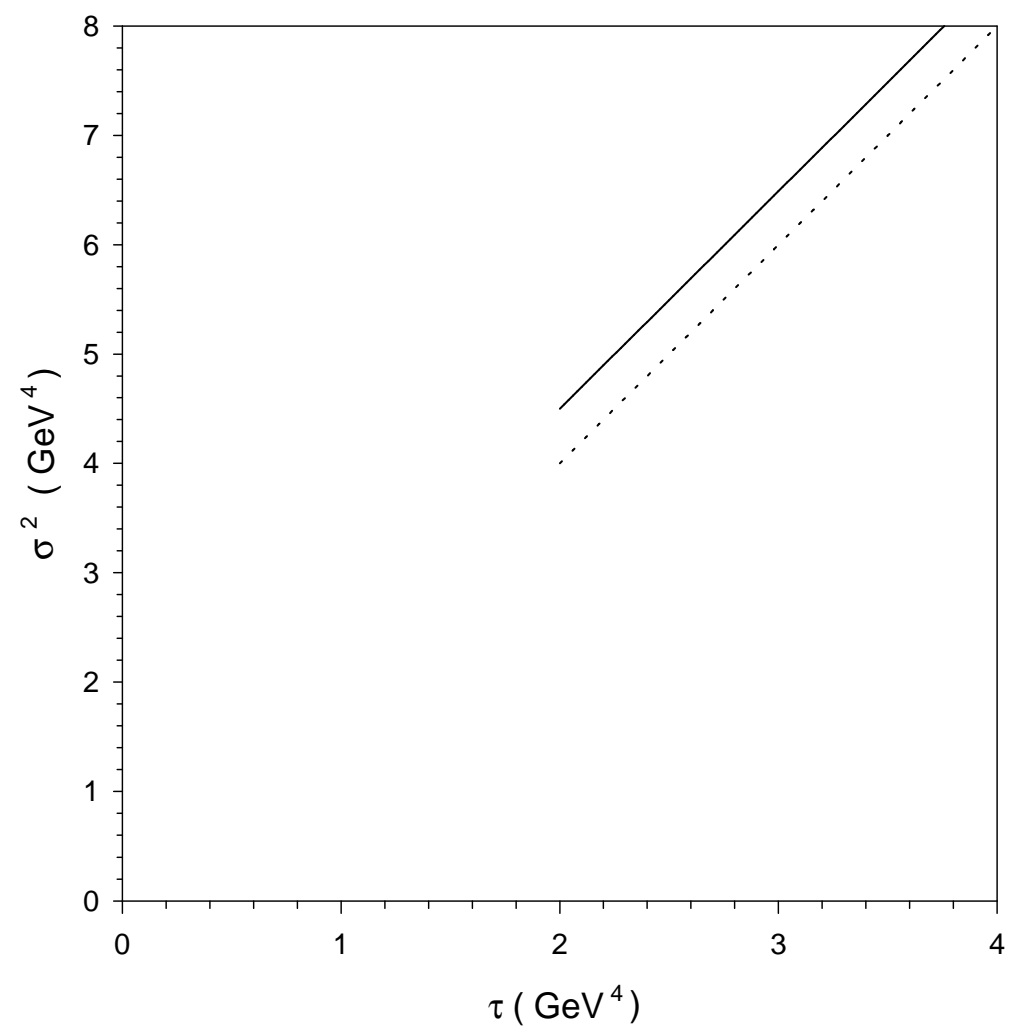

Figure 7: Plot of $\sigma^{2}$ for the theoretical prediction (solid curve) compared with $\sigma^{2}=2 \tau$ for the single-resonance model (dashed curve) for the $I=1$ scalar channel using the $\chi^{2}$-optimized value of the continuum $s_{0}=3.9 \mathrm{GeV}^{2}$. 


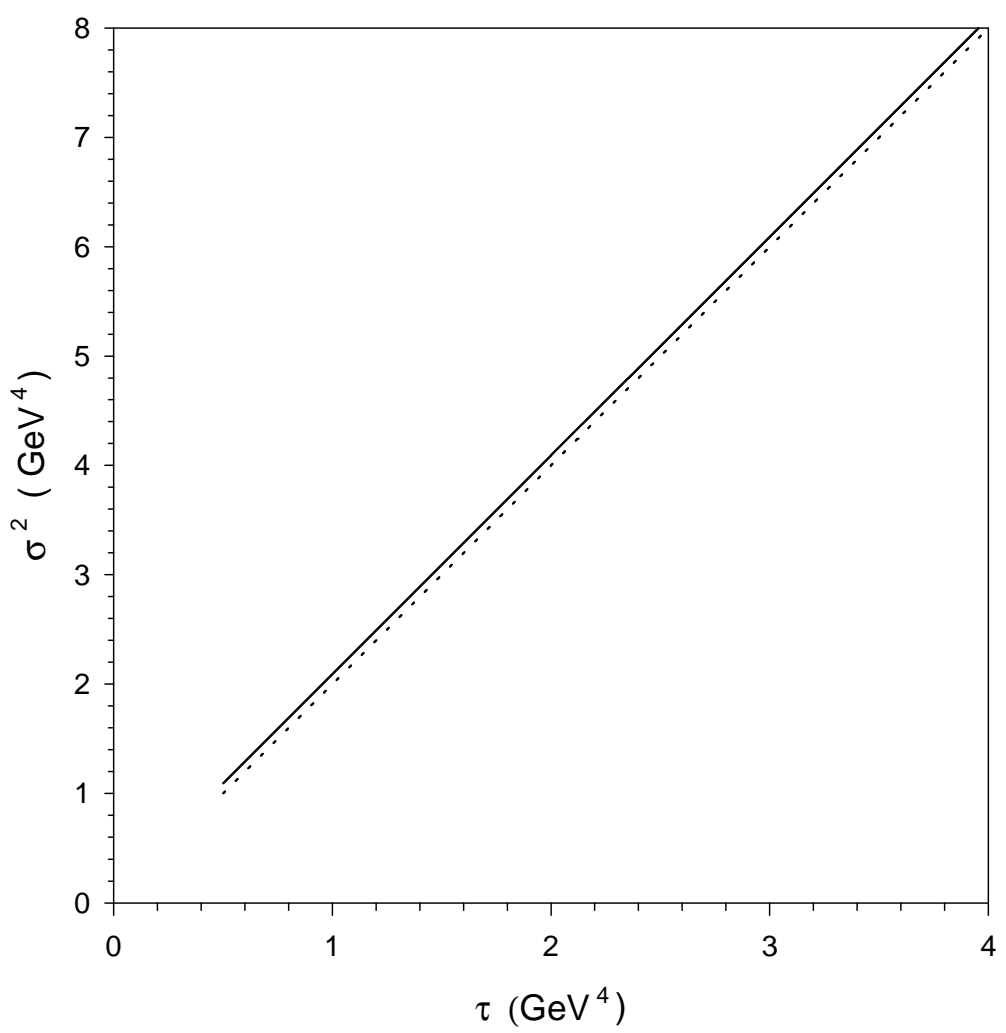

Figure 8: Plot of $\sigma^{2}$ for the theoretical prediction (solid curve) compared with $\sigma^{2}=2 \tau$ for the single-resonance model (dashed curve) for the vector channel using the $\chi^{2}$-optimized value of the continuum $s_{0}=1.22 \mathrm{GeV}^{2}$. 


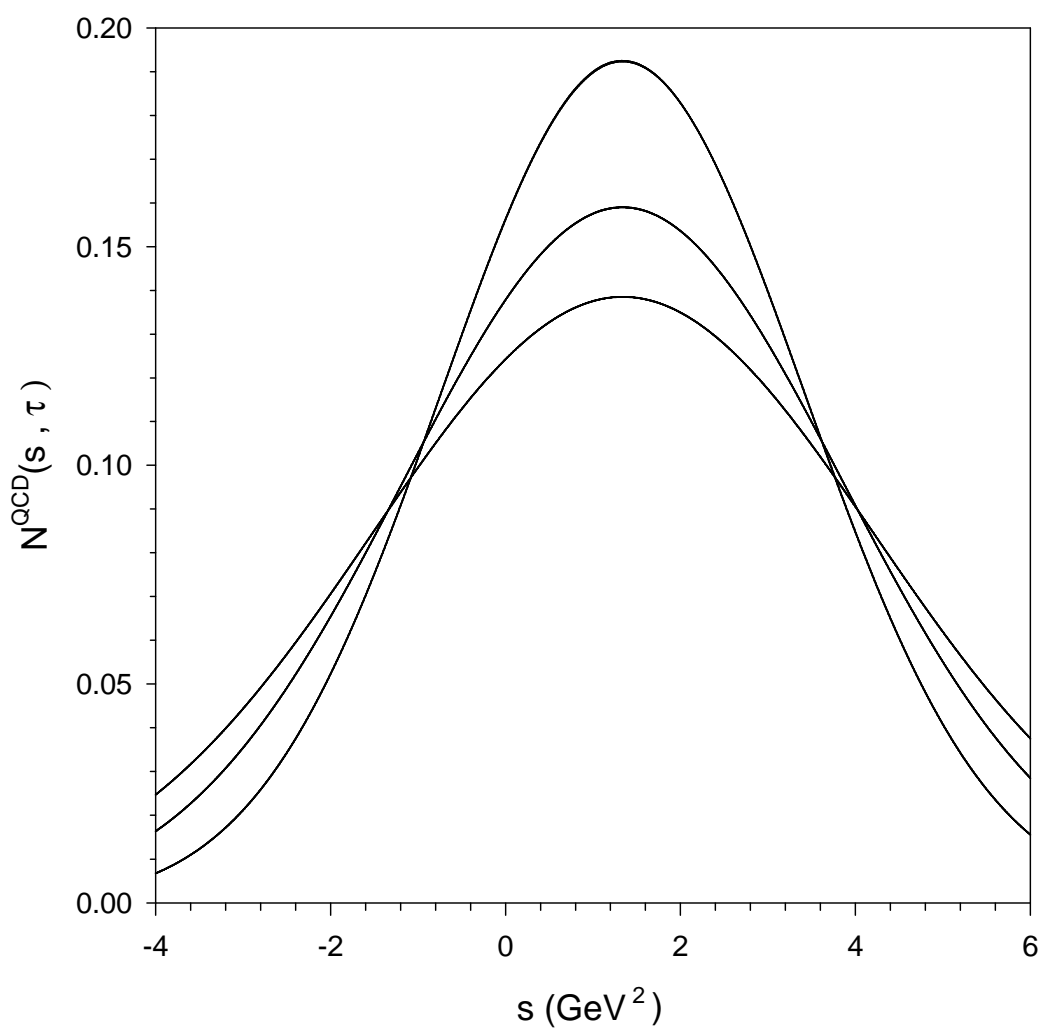

Figure 9: Comparison of the $I=0$ scalar-current theoretical prediction $N^{Q C D}\left(\hat{s}, \tau, s_{0}\right)$ with the two-resonance phenomenological model using the $\chi^{2}$-optimized values of the resonance masses $\left(m_{1}=0.97 \mathrm{GeV}, m_{2}=1.43 \mathrm{GeV}\right)$, relative resonance strengths $\left(r_{1}=0.634, r_{2}=0.366\right)$ and continuum $s_{0}=2.35 \mathrm{GeV}^{2}$. The $\tau$ values used for the four pairs of curves, from top to bottom in the figure, are respectively $\tau=2.0 \mathrm{GeV}^{4}, \tau=3.0 \mathrm{GeV}^{4}$, and $\tau=4.0 \mathrm{GeV}^{4}$. Note the almost perfect overlap between the theoretical prediction and phenomenological model. Central values of the condensate parameters have been used. 


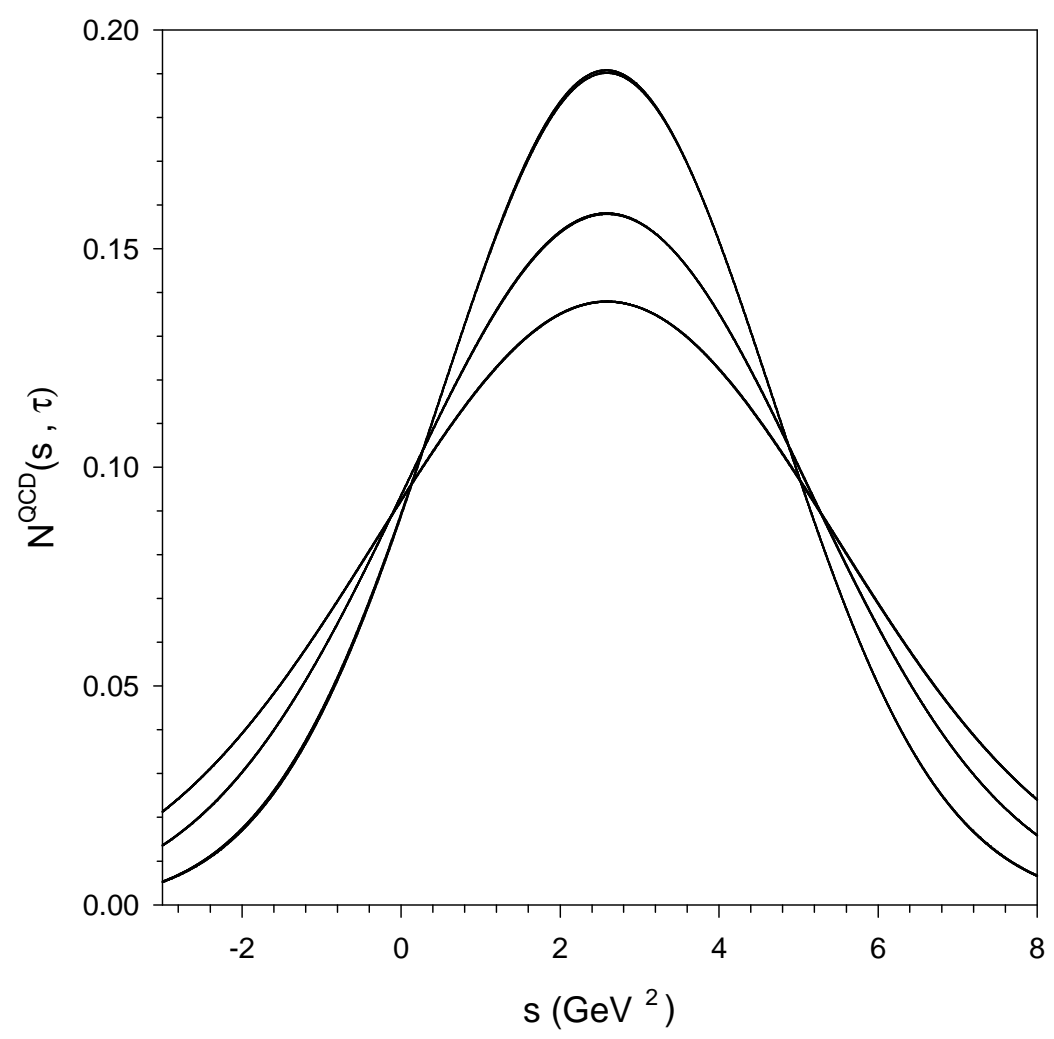

Figure 10: Comparison of the $I=1$ scalar current theoretical prediction $N^{Q C D}\left(\hat{s}, \tau, s_{0}\right)$ with the two-resonance phenomenological model using the $\chi^{2}$-optimized values of the resonance masses $\left(m_{1}=1.44 \mathrm{GeV}, m_{2}=1.81 \mathrm{GeV}\right)$, relative resonance strengths $\left(r_{1}=0.570, r_{2}=0.430\right)$ and continuum $s_{0}=3.50 \mathrm{GeV}^{2}$. The $\tau$ values used for the four pairs of curves, from top to bottom in the figure, are respectively $\tau=2.0 \mathrm{GeV}^{4}, \tau=3.0 \mathrm{GeV}^{4}$, and $\tau=4.0 \mathrm{GeV}^{4}$. Note the almost perfect overlap between the theoretical prediction and phenomenological model. Central values of the condensate parameters have been used. 